\title{
Effect of Heat Source and Imperfect Contact on Simultaneous Estimation of Thermal Properties of High-Conductivity Materials
}

\author{
Giampaolo D’Alessandro $\mathbb{D}^{1},{ }^{1}$ Filippo de Monte ${ }^{\mathbb{D}},{ }^{1}$ and Donald E. Amos ${ }^{2}$ \\ ${ }^{1}$ Department of Industrial and Information Engineering and Economics, University of L'Aquila, via G. Gronchi 18, \\ 67100 L'Aquila, Italy \\ ${ }^{2}$ Sandia National Laboratories, Albuquerque, NM 87110, USA
}

Correspondence should be addressed to Giampaolo D’Alessandro; giampaolo.dalessandro@univaq.it

Received 27 October 2018; Revised 26 February 2019; Accepted 2 May 2019; Published 23 June 2019

Academic Editor: Michael Vynnycky

Copyright (c) 2019 Giampaolo D’Alessandro et al. This is an open access article distributed under the Creative Commons Attribution License, which permits unrestricted use, distribution, and reproduction in any medium, provided the original work is properly cited.

In the current paper a novel methodology accounting for both the heater heat capacity and the imperfect thermal contact between a thin heater and a specimen is proposed. In particular, the volumetric heat capacity of the heater is considered by modelling it as a lumped capacitance body, while the imperfect thermal contact is considered by means of a contact resistance. Thus, the experimental apparatus consisting of three layers (specimen-heater-specimen) is reduced to a single finite layer (sample) subject to a "nonclassical" boundary condition at the heated surface, known as sixth kind. Once the temperature solution is derived analytically using the Laplace transform method, the scaled sensitivity coefficients are computed analytically at the interface between the heater and the sample (heater side and sample side) and at the sample backside. By applying the proposed methodology to a lab-controlled experiment available in the specialized literature, a reduction of the thermal properties values of about $1.4 \%$ is observed for a highconductivity material (Armco iron).

\section{Introduction}

Sensitivity coefficients are used in parameter estimation [1], optimal experimental design [2], and uncertainty analysis [3]. In parameter estimation, a sensitivity analysis of the temperature to the unknown parameters, such as thermal conductivity, volumetric heat capacity, or thermal diffusivity, provides valuable insight. For example, in an experimental apparatus for which sensitivity coefficients had not been studied adequately it is possible that additional materials used in the experimental set-up (for instance, the thin heater giving up heat to the sample) may affect the temperature more than the material under investigation (sample); also, the temperature sensor location might not be the optimal one. Even the uncertainty in the profile of the applied heat flux may represent a mode of noise in the experiment [4]; in such a case an analysis of the temperature sensitivity to the applied heat flux is needed. All these features can compromise the quality of the results [2].
Also, the sensitivity coefficients not only make possible a preliminary evaluation of the goodness of the experimental results (at least from a qualitative point of view), but are also directly involved in the estimation of the parameters by means of the sensitivity matrix when minimizing the ordinary least square norm [5-7] whose convergence difficulties may be predicted by establishing whether linear dependence between sensitivity coefficients exists [4].

As known, the parameter estimation technique requires measured temperature values obtained by transient [8] measurements. One of the principles of parameter estimation is that the measured temperature values are sensitive to the parameters of interest: the more sensitive the temperature (or large the sensitivity coefficient) is, the more valuable the temperature measurements are $[2,8]$, though the accuracy of the estimates may adversely be affected by a large magnitude difference between the same coefficients [4]. Furthermore, the sensitivity coefficients have to be uncorrelated; otherwise it is impossible to estimate two or more parameters simultaneously $[2,8]$. 


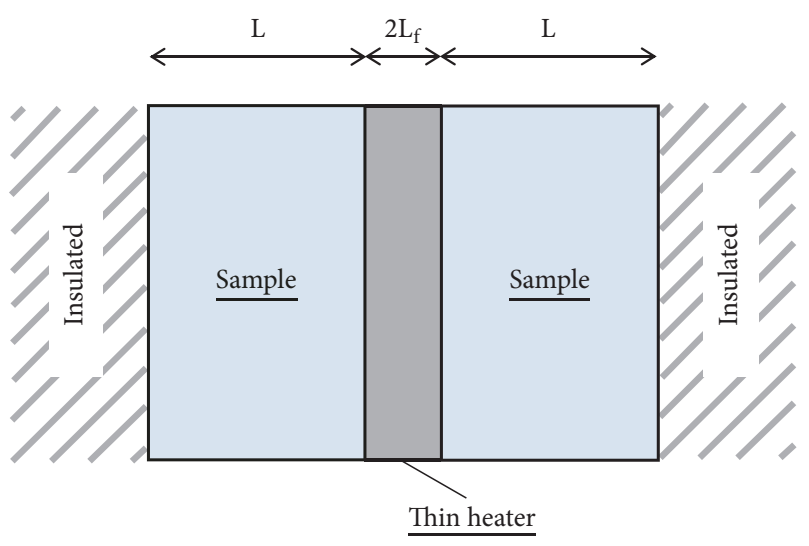

(a)

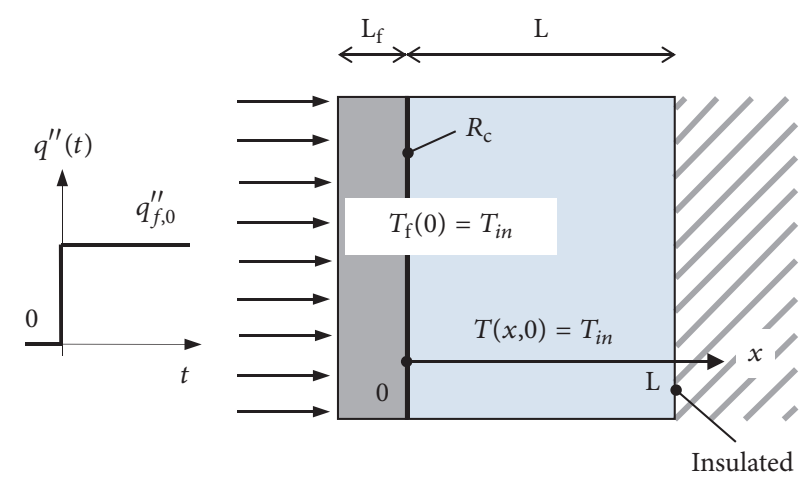

(b)

FIGURE 1: Schematic of the experimental apparatus for thermal properties measurements (a) and simplified schematic for the addressed problem (b).

The focus of the current paper is to develop a new procedure accounting for both the heater heat capacity and the imperfect thermal contact between the thin heater and the specimen. For this reason, the dependence of the thermal properties on temperature is not considered here. Research work regarding the estimation of temperature-dependent properties is available in literature as Refs. [7, 9]. Then, if the thin heater can be modelled as a lumped capacitance body, the addressed transient heat conduction problem concerns a one dimensional (1-D) finite single-layer rectangular body (sample) subject to a boundary condition of the sixth kind at the heated boundary and insulated at the backside. The boundary condition of the 6th kind is somewhat neglected in the specialized heat conduction literature with the exception of Refs. [10, p. 22], [11]. Note that the word sixth kind is not used in Ref. [10], while it is used in [11, p. 160].

The governing equations are conveniently solved by using the Laplace Transform method. In particular, the solution calculated in the Laplace domain is inverted by means of the residues theorem which requires the computation of the poles and of the corresponding residues. Once the thermal field is obtained, the so-called "scaled" sensitivity coefficients are computed analytically by performing the partial derivative of the temperature with respect to the parameters of interest. The coefficients are presented in a graphical form for three different locations: (1) at the interface between the heater and the sample (heater side), (2) at the interface between the heater and the sample (sample side), and (3) at the sample back side. A discussion about the better locations of the temperature sensors is also given.

The results of the proposed procedure are then applied to a lab-controlled experiment available in the parameter estimation literature [8, Chap. 7]. By comparing measured and calculated temperatures through the ordinary least squares norm and, then, minimizing this by the well-established Gauss method, a reduction of about $1.4 \%$ was observed for the thermal properties values of the sample considered (Armco iron). This slight reduction is however in accordance with the very thin heater and the very low contact resistance of the experimental apparatus considered.

\section{Mathematical Formulation}

A schematic of the experimental apparatus for the measurement of the thermal properties of a solid specimen is depicted in Figure 1(a). This figure shows a thin layer heater (metallic or kapton/silicon) located between two highconductivity samples of the same material and thickness which releases heat at the surfaces of both samples. This threelayer configuration (specimen-heater-specimen) reduces to the simplified configuration depicted in Figure 1(b) by virtue of a thermal symmetry. In particular, the finite body is in imperfect thermal contact with a thin layer (representing the heater as a lumped body) at the boundary $x=0$ through a surface contact resistance $R_{c}$. In particular, this resistance increases when using metallic resistive heaters (made of copper or aluminium) as they are rigid, while it decreases if kapton or silicon resistive heaters are utilized as they are flexible. Then, the finite body and the thin layer are at the same uniform initial temperature $T_{\text {in }}$. Also, at $t=0$ a step change in the surface heat flux is applied to the thin layer, whose thickness and volumetric heat capacity are denoted by $L_{\mathrm{f}}$ and $C_{\mathrm{f}}$, respectively. In addition, the finite body is insulated at the backside $x=L$, and its properties $k$ and $C$ are considered temperature-independent, as is $C_{f}$.

The mathematical formulation of this transient, linear, 1$\mathrm{D}$ heat conduction problem is defined as

$$
\frac{\partial^{2} T}{\partial x^{2}}=\frac{1}{\alpha} \frac{\partial T}{\partial t}
$$

$$
(0<x<L ; t>0)
$$

$$
\begin{aligned}
-k\left(\frac{\partial T}{\partial x}\right)_{x=0}+L_{f} C_{f} \frac{d T_{f}}{d t}=q_{f, 0}^{\prime \prime} \quad(t>0) \\
-k\left(\frac{\partial T}{\partial x}\right)_{x=0}=\frac{1}{R_{c}}\left[T_{f}(t)-T(0, t)\right]
\end{aligned}
$$




$$
\begin{array}{rlrl}
-k\left(\frac{\partial T}{\partial x}\right)_{x=L} & =0 & (t>0) \\
T(x, 0) & =0 \quad(0<x<L) \\
T_{\mathrm{f}}(0) & =0
\end{array}
$$

where Eqs. (1b) and (1c) represent the boundary condition of the sixth kind at $x=0$. In particular, the former equation is obtained by applying the first law of thermodynamics to the thin layer assumed as a lumped-capacitance body, where $q_{\mathrm{f}, 0}^{\prime \prime}=g_{\mathrm{f}, 0} L_{\mathrm{f}}\left(g_{\mathrm{f}, 0}\right.$ denotes the volumetric heat generation within the heater), while the latter accounts for the imperfect contact between the thin layer and the finite body. Also, the perfect insulated condition presented in Eq. (1d) is consistent only with high-thermal conductivity samples.

Then, according to the numbering system devised in [12, Chap. 2], [13], the above problem may be denoted by X62B10T11. In particular, this number denotes a transient heat conduction problem concerning a $1 \mathrm{D}$ rectangular finite body (by the $\mathrm{X}$ ), subject to a boundary condition of the sixth kind at the surface $x=0$ (by the 6 in X62) with a constant applied heat flux (by the B1), and having an insulated boundary at $x=L$ (type 2 boundary condition by the 2 in X62, and zero heat flux by the 0 in B10); also, T11 denotes a nonzero uniform initial temperature for both layers.

By using the following dimensionless variables,

$$
\begin{aligned}
\widetilde{T} & =\frac{T-T_{\mathrm{in}}}{q_{\mathrm{f}, 0}^{\prime \prime} L / k}, \\
\tilde{x} & =\frac{x}{L}, \\
\tilde{t} & =\frac{\alpha t}{L^{2}}, \\
\widetilde{T}_{\mathrm{f}} & =\frac{T_{\mathrm{f}}-T_{\mathrm{in}}}{q_{\mathrm{f}, 0}^{\prime \prime} L / k}, \\
P & =\frac{C_{\mathrm{f}} L_{\mathrm{f}}}{C L}, \\
\widetilde{R}_{\mathrm{c}} & =\frac{R_{\mathrm{c}}}{L / k}
\end{aligned}
$$

the governing equations given by Eqs. (1a)-(1f), in dimensionless form, become

$$
\begin{aligned}
& \frac{\partial^{2} \widetilde{T}}{\partial \widetilde{x}^{2}}=\frac{\partial \widetilde{T}}{\partial \widetilde{t}} \quad(0<\tilde{x}<1 ; \tilde{t}>0) \\
&-\left(\frac{\partial \widetilde{T}}{\partial \widetilde{x}}\right)_{\tilde{x}=0}+P \frac{\partial \widetilde{T}_{\mathrm{f}}}{\partial \widetilde{t}}=1 \quad(\tilde{t}>0) \\
&-\left(\frac{\partial \widetilde{T}}{\partial \widetilde{x}}\right)_{\tilde{x}=0}=\frac{1}{\widetilde{R}_{\mathrm{c}}}\left[\widetilde{T}_{\mathrm{f}}(\tilde{t})-\widetilde{T}(0, \tilde{t})\right] \\
&-\left(\frac{\partial \widetilde{T}}{\partial \widetilde{x}}\right)_{\tilde{x}=1}=0 \quad(\widetilde{t}>0)
\end{aligned}
$$$$
(\tilde{t}>0)
$$

$$
\begin{aligned}
\widetilde{T}(\tilde{x}, 0) & =0 \quad(0<\tilde{x}<1) \\
\widetilde{T}_{\mathrm{f}}(0) & =0
\end{aligned}
$$

Moreover, as the initial conditions are now homogeneous, Eqs. (3e) and (3f), the problem notation becomes X62B10T00.

\section{Temperature Solution}

The temperature solution may be derived by applying the Laplace Transform method to the governing equations, Eqs. (3a)-(3f), as shown below. In fact, taking into account the initial conditions Eqs. (3e) and (3f), Eqs. (3a)-(3f) yield

$$
\begin{aligned}
\frac{\partial^{2} \vartheta}{\partial \tilde{x}^{2}} & =s \vartheta \quad(0<\tilde{x}<1) \\
-\left.\frac{\partial \vartheta}{\partial \tilde{x}}\right|_{\tilde{x}=0}+P s \vartheta_{f} & =\frac{1}{s} \\
\vartheta_{f} & =\vartheta(0, s)-\left.\widetilde{R}_{c} \frac{\partial \vartheta}{\partial \tilde{x}}\right|_{\tilde{x}=0} \\
\left(\frac{\partial \vartheta}{\partial \tilde{x}}\right)_{\tilde{x}=1} & =0
\end{aligned}
$$

where $\vartheta(\tilde{x}, s)=L[\widetilde{T}(\tilde{x}, \tilde{t})]$.

The solution of the problem defined by Eqs. (4a)-(4d) results in

$$
\begin{aligned}
\vartheta(\tilde{x}, q) & =B_{1} \cosh (q \tilde{x})+B_{2} \sinh (q \tilde{x}) \\
& =A_{1} \cosh (q \tilde{x}+\varphi)
\end{aligned}
$$

where $q=\sqrt{s}$ and the phase $\varphi$ can be determined by applying the boundary condition at $\tilde{x}=1$, i.e., Eq. (4d). It follows that $\varphi=-q$.

Also, it is possible to determine the constant $A_{1}$ by using the boundary condition of the sixth kind defined by Eqs. (4b) and (4c) in the Laplace domain. Then, bearing in mind that the hyperbolic cosine is an even function, the sought solution $\vartheta(\widetilde{x}, q)$ is

$$
\begin{aligned}
\vartheta(\tilde{x}, q) & =\frac{\cosh [q(1-\tilde{x})]}{\underbrace{q^{2}\left[P q^{2} \cosh (q)+q\left(P \widetilde{R}_{c} q^{2}+1\right) \sinh (q)\right]}_{D(q)}} \\
& =\frac{\cosh [q(1-\tilde{x})]}{D(q)}
\end{aligned}
$$

Now a Taylor series expansion of the hyperbolic functions allows us to gain insight on the poles of the solution defined by Eq. (6). Hence, the function $D(q)$ appearing at the denominator of the above equation becomes

$$
\begin{aligned}
& D(q)=q^{2}\left\{P q^{2}\left[1+\frac{q^{2}}{2}+O\left(q^{4}\right)\right]\right. \\
& \left.+q\left(P \widetilde{R}_{c} q^{2}+1\right)\left[q+\frac{q^{3}}{3 !}+O\left(q^{5}\right)\right]\right\}=q^{4}\{P+1 \\
& \left.+q^{2}\left[\frac{P}{2}+P \widetilde{R}_{c}+\frac{1}{6}\right]+O\left(q^{4}\right)\right\}
\end{aligned}
$$


Therefore, this function admits a quadruple pole at $q=0$ (which implies a double pole at $s=0$ ). Also, it has simple zeros along the negative real axis that may conveniently be assumed as $q=i \beta$ (or $s=-\beta^{2}$ ). Then, the value of $\beta$ can be computed by solving numerically the eigencondition $D(q=i \beta)=0$; that is,

$$
P \beta \cos (\beta)+\left(1-P \widetilde{R}_{c} \beta^{2}\right) \sin (\beta)=0
$$

where the relations $\cosh (i \beta)=\cos (\beta)$ and $\sinh (i \beta)=i \sin (\beta)$ have been used. Eq. (8) admits an infinite number of simple poles at $q=i \beta_{m}, \beta_{m}>0, m=1,2, \ldots$ (or $s=-\beta_{m}^{2}$ ), where $\beta_{m}$ is the $m$-th eigenvalue whose computation is discussed in Section 3.1.

Since the function $\vartheta(\tilde{x}, q)$ in Eq. (8) is analytic except at poles $q=0(s=0)$ and at poles $q=i \beta_{m}\left(s=-\beta_{m}^{2}\right), \beta_{m}>0$, $m=1,2$..., the inverse of such a function may be obtained using the residue theorem [14, pp. 384-385]

$$
\begin{aligned}
\widetilde{T}(\tilde{x}, \tilde{t}) & =L^{-1}\{\vartheta(\tilde{x}, s)\}=\frac{1}{2 \pi i} \int_{\gamma-i \infty}^{\gamma+i \infty} \vartheta(\tilde{x}, s) e^{s t} d s \\
& =\sum_{\text {encompassing the left half plane }}^{\text {Residues in the closed contour }} \\
& =R_{s=0}+\sum_{m=1}^{\infty} R_{s=-\beta_{m}^{2}}
\end{aligned}
$$

where the calculation of the residues, $R_{s=0}$ and $R_{s=-\beta_{m}^{2}}$, is given in the Appendix. In particular, they result in

$$
\begin{aligned}
& R_{s=0}=\frac{\widetilde{t}}{P+1}+\frac{\widetilde{x}^{2}}{2(P+1)}-\frac{\tilde{x}}{P+1}+\frac{1-3 P \widetilde{R}_{c}}{3(P+1)^{2}} \\
& R_{s=-\beta_{m}^{2}} \\
& =\frac{2\left(P \widetilde{R}_{c} \beta_{m}^{2}-1\right) \cos \left[\beta_{m}(1-\widetilde{x})\right] e^{-\beta_{m}^{2} \widetilde{t}}}{\left[\left(P \widetilde{R}_{c}\right)^{2} \beta_{m}^{4}+\left(P+P \widetilde{R}_{c}-2 \widetilde{R}_{c}\right) P \beta_{m}^{2}+P+1\right] \beta_{m}^{2} \cos \left(\beta_{m}\right)}
\end{aligned}
$$

Substituting Eqs. (10a) and (10b) in Eq. (9) yields the temperature solution in the time domain.

$$
\begin{aligned}
& \widetilde{T}(\tilde{x}, \tilde{t}) \\
& =\frac{\tilde{t}}{P+1}+\frac{\tilde{x}^{2}}{2(P+1)}-\frac{\tilde{x}}{P+1}+\frac{1-3 P \widetilde{R}_{c}}{3(P+1)^{2}} \\
& \quad+2 \sum_{m=1}^{\infty} \frac{\left(P \widetilde{R}_{c} \beta_{m}^{2}-1\right) \cos \left[\beta_{m}(1-\tilde{x})\right]}{\widetilde{N}_{m} \beta_{m}^{2} \cos \left(\beta_{m}\right)} e^{-\beta_{m}^{2} \tilde{t}} \\
& \quad(0 \leq \tilde{x} \leq 1 ; \tilde{t} \geq 0)
\end{aligned}
$$

where $\widetilde{N}_{m}$ is the dimensionless norm given by

$$
\widetilde{N}_{m}=\left(P \widetilde{R}_{c}\right)^{2} \beta_{m}^{4}+\left(P+P \widetilde{R}_{c}-2 \widetilde{R}_{c}\right) P \beta_{m}^{2}+P+1
$$

Once $\widetilde{T}(\tilde{x}, \widetilde{t})$ is known, the thin layer temperature $\widetilde{T}_{f}(\widetilde{t})$ may be obtained by using the interface condition defined by the latter of Eq. (3b). After some algebra, it is given by

$$
\widetilde{T}_{f}(\tilde{t})=\frac{\tilde{t}}{P+1}+\frac{\widetilde{R}_{c}+1 / 3}{(P+1)^{2}}-2 \sum_{m=1}^{\infty} \frac{e^{-\beta_{m}^{2} \tilde{t}}}{\widetilde{N}_{m} \beta_{m}^{2}} \quad(\tilde{t} \geq 0)
$$

3.1. Computation of the Eigenvalues. The eigencondition defined by Eq. (8) may be rewritten as

$$
\tan \left(\beta_{m}\right)=\frac{\beta_{m} / \widetilde{R}_{\mathrm{c}}}{\beta_{m}^{2}-1 /\left(P \widetilde{R}_{\mathrm{c}}\right)}
$$

It is worth noting that Eq. (12) is similar to the corresponding equation of the X33 case treated in [15], where "X33" denotes a $1 \mathrm{D}$ rectangular finite body with boundary conditions of the third kind on both sides (assuming $B i_{1}$ at $x=0$ and $B i_{2}$ at $x=L$ ). In fact, it is sufficient to replace the sum of the Biot numbers $\left(B i_{1}+B i_{2}\right)$ with the term $1 / \widetilde{R}_{c}$, and their product $\left(B i_{1} B i_{2}\right)$ with the term $1 /\left(P \widetilde{R}_{c}\right)$. Therefore, its roots may be computed by using the same explicit approximate relations based on the third-order modified Newton method [15]. In particular, the approximate eigenvalue $\zeta_{m}$ is obtained by using an iteration process as shown below:

$$
\beta_{m} \approx \zeta_{m}=z_{m}+\varepsilon_{m}\left(z_{m}\right)
$$

where $\beta_{m}$ is the exact eigenvalue, $z_{m}$ is the initial guess value used in the first iteration, and $\varepsilon_{m}$ is the deviation which yields the update value for $\zeta_{m}$.

These relations provide an approximate value of the exact eigenvalue with high accuracy (to at least 7 th decimal place when $m=1$, and a higher decimal place for $m>1$, after one iteration) for $P \in(0, \infty)$ and $\widetilde{R}_{c} \in(0, \infty)$. To obtain eigenvalues with an accuracy of $10^{-15}$ two more iterations may be required.

In particular, a computer code called "fdeigX62" for calculating these eigenvalues is available in Ref. [16, App. B] for ease of use. Some of them computed for $P=\widetilde{R}_{c}=1$ are shown in Table 1 , where the exact eigenvalues $\beta_{m}$ listed in the last column are obtained by using the internal Matlab function "fsolve" setting the tolerance parameter equal to $10^{-15}$. Note that $\zeta_{m}$ after two iterations is exactly the same as $\beta_{m}$, but $\beta_{m}$ is obtained after three iterations starting from the same initial guess $z_{m}$.

3.2. Computational Solution. The exact analytical solutions given by Eqs. (11a) and (11c) exhibit an infinite series, while the computational solution requires a finite number of terms, 
TABLE 1: Comparison of the first ten calculated $\zeta_{m}$ and exact $\beta_{m}$ eigenvalues for the X62 case.

\begin{tabular}{|c|c|c|c|c|}
\hline$m$ & $\begin{array}{c}z_{m} \\
\text { (initial guess) }\end{array}$ & $\begin{array}{c}\zeta_{m} \\
\text { (after 1 iteration) }\end{array}$ & $\begin{array}{c}\zeta_{m} \\
\text { (after } 2 \text { iterations) }\end{array}$ & $\begin{array}{c}\beta_{m} \\
\text { (exact) }\end{array}$ \\
\hline 1 & 1.195476244024383 & $1.207792 \underline{438624699}$ & 1.207792655153241 & 1.207792655153241 \\
\hline 2 & 3.486925937947245 & $3.4482 \underline{42071207367}$ & 3.448237983351985 & 3.448237983351985 \\
\hline 3 & 6.468623593104900 & $6.44095 \underline{6097445878}$ & 6.440954447908016 & 6.440954447908016 \\
\hline 4 & 9.550944741970163 & $9.530477 \underline{847514561}$ & 9.530477156207574 & 9.530477156207574 \\
\hline 5 & 12.662238896069196 & $12.64577 \underline{9030853410}$ & 12.645778666218552 & 12.645778666218552 \\
\hline 6 & 15.785462794465527 & $15.771538 \underline{985089661}$ & 15.771538762863299 & 15.771538762863299 \\
\hline 7 & 18.914707557510582 & $18.902557 \underline{753172037}$ & 18.902557604969395 & 18.902557604969395 \\
\hline 8 & 22.047411709862786 & 22.036590088566303 & 22.036589983609232 & 22.036589983609232 \\
\hline 9 & 25.182288796593070 & $25.1725089 \underline{98808841}$ & 25.172508921226136 & 25.172508921226136 \\
\hline 10 & 28.318621989478178 & 28.309686934070488 & 28.309686874845468 & 28.309686874845468 \\
\hline
\end{tabular}

say $m_{\max }$ terms. Therefore, a convergence criterion for the series solutions is needed and it is defined as follows:

$$
\varepsilon=\frac{\left|\widetilde{T}_{c}(\tilde{x}, \widetilde{t}, A)-\widetilde{T}(\tilde{x}, \tilde{t})\right|}{\widetilde{T}_{f}(\tilde{t})}=\frac{2\left|\sum_{m=m_{\max }+1}^{\sum_{m}^{\infty}\left\{\left(P \widetilde{R}_{c} \beta_{m}^{2}-1\right) \cos \left[\beta_{m}(1-\tilde{x})\right] / \widetilde{N}_{m} \beta_{m}^{2} \cos \left(\beta_{m}\right)\right\} e^{-\beta_{m}^{2} \tilde{t}}}\right|}{\widetilde{t} /(P+1)+\left(\widetilde{R}_{c}+1 / 3\right) /(P+1)^{2}-2 \sum_{m=1}^{\infty}\left(e^{-\beta_{m}^{2} \tilde{t}} / \widetilde{N}_{m} \beta_{m}^{2}\right)} \leq 10^{-A}
$$

where $\widetilde{T}(\tilde{x}, \tilde{t})$ denotes the exact solution (with an infinite number of terms), $\widetilde{T}_{c}(\tilde{x}, \tilde{t}, A)$ represents the computational one with a finite number of terms $m_{\max }$ for a truncation error $\varepsilon$ of $10^{-A}(A=2,3, \ldots 15)$, and $\widetilde{S}_{T}$ is the "tail" of the series solutions that may be calculated by using the Euler-McLaurin formula [17]. Then, by solving numerically Eq. (14) for $m_{\max }$ and by fitting properly the obtained numerical data, the maximum number of terms may be taken in a conservative way as

$$
\begin{aligned}
& m_{\max } \\
& =1 \\
& \quad+\operatorname{ceil}\left\{\frac{1}{\pi} \sqrt{\frac{A \ln (10)+[H(P-1)-1] \ln (P)}{\widetilde{t}}}\right\}
\end{aligned}
$$

where $H(\cdot)$ denotes the Heaviside step function, while "ceil $(z)$ " is a Matlab function that rounds the number $z$ to the nearest integer greater than or equal to $z$.

It is worth noting that $m_{\max }$ is independent of both the thermal contact resistance $\widetilde{R}_{c}$ and the location $\tilde{x}$. When $P>1$, it is independent of $P$ too.

According to Eq. (15), a large number of terms may be required for early times. Therefore, for times less than the socalled deviation time $\widetilde{t}_{\mathrm{d}}$ defined as $[13,18]$

$$
\tilde{t}_{\mathrm{d}}=\frac{1}{10 A}(2-\tilde{x})^{2}
$$

the temperature solution can be replaced by a semi-infinite transient solution available in Ref. [19] with an error less than $10^{-A}(A=2,3, \ldots 15)$, where an intrinsic verification of the solution was proven. A computer code in Matlab ambient for calculating the solution of the current X62B10T00 problem is provided in [16, App. E] for ease of use. A plot of dimensionless temperature and heat flux as a function of time at the heated surface of the sample for different $P$ values and $\widetilde{R}_{\mathrm{c}}=1$ is given in Figure 2 . It is evident that the heat flux at $\tilde{x}=0$ is time-dependent and this dependence is due to both the thermal inertia of the heat source and the contact resistance.

\section{Sensitivity Coefficients}

The scaled sensitivity coefficients, that is, the partial derivatives of the temperature with respect to the model parameter $\gamma$ of interest (e.g., $q_{\mathrm{f}, 0}^{\prime \prime}, k, C, C_{\mathrm{f}}, R_{\mathrm{c}}$ ) multiplied by the parameter itself, are defined as follows:

$$
\begin{gathered}
X_{\gamma}=\gamma \frac{\partial T}{\partial \gamma} \\
X_{\gamma, \mathrm{f}}=\gamma \frac{\partial T_{\mathrm{f}}}{\partial \gamma}
\end{gathered}
$$

Therefore, as suggested by the above equation the scaled sensitivity coefficients have units of ${ }^{\circ} \mathrm{C}$ and their calculation 


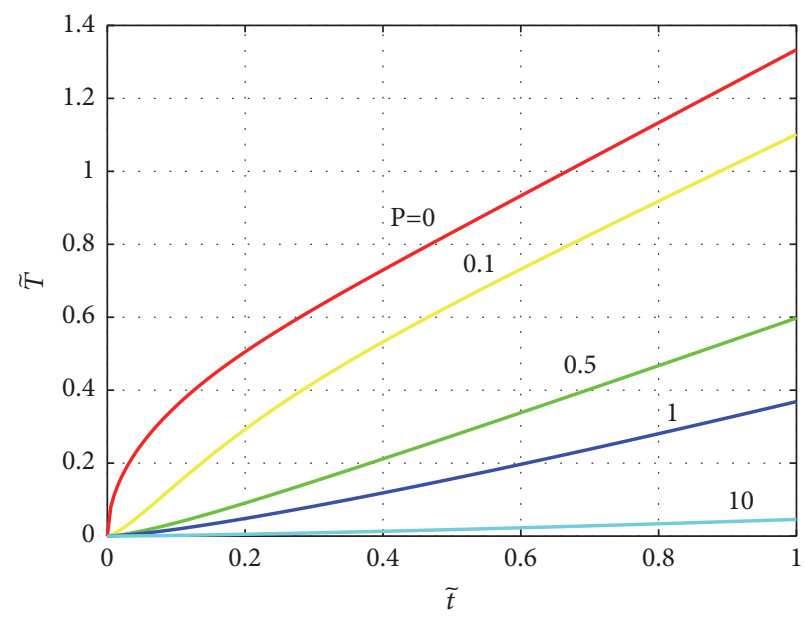

(a)

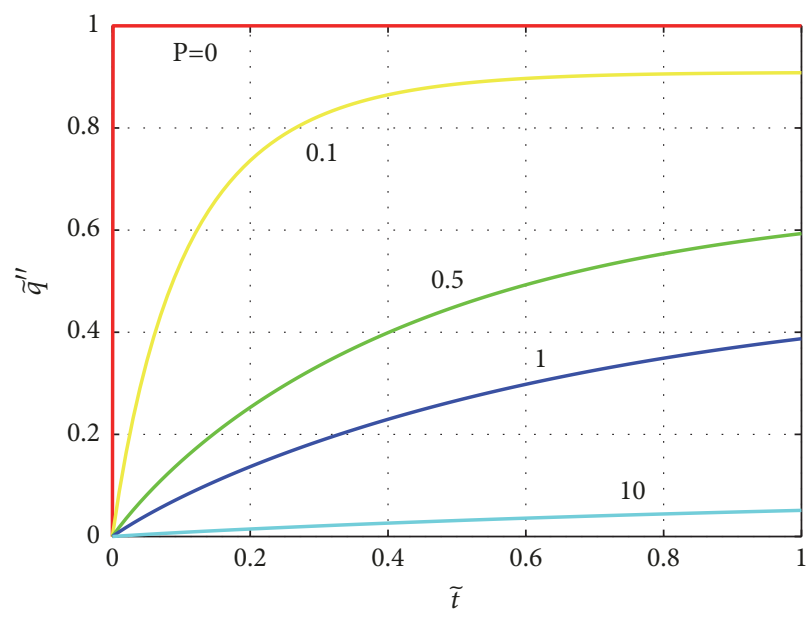

(b)

Figure 2: Dimensionless temperature (a) and heat flux (b) as a function of the dimensionless time at $\widetilde{x}=0$, for different $P$ values and $\widetilde{R}_{\mathrm{c}}=1$.

requires the use of the chain rule [20]. In dimensionless form they become

$$
\begin{aligned}
\widetilde{X}_{\gamma} & =\frac{X_{\gamma}}{q_{\mathrm{f}, 0}^{\prime \prime} L / k}, \\
\widetilde{X}_{\gamma, \mathrm{f}} & =\frac{X_{\gamma, \mathrm{f}}}{q_{\mathrm{f}, 0}^{\prime \prime} L / k}
\end{aligned}
$$

4.1. Heat Flux as a Parameter. If the parameter of interest is the applied heat flux at the boundary $\tilde{x}=0$, the corresponding sensitivity coefficient is

$$
\begin{aligned}
X_{q_{\mathrm{f}, 0}^{\prime \prime}} & =q_{\mathrm{f}, 0}^{\prime \prime} \frac{\partial T}{\partial q_{\mathrm{f}, 0}^{\prime \prime}}=q_{\mathrm{f}, 0}^{\prime \prime} \frac{\partial}{\partial q_{\mathrm{f}, 0}^{\prime \prime}}\left(\widetilde{T} \frac{q_{\mathrm{f}, 0}^{\prime \prime} L}{k}\right) \\
& =q_{\mathrm{f}, 0}^{\prime \prime}[\frac{q_{\mathrm{f}, 0}^{\prime \prime} L}{k} \underbrace{\frac{\partial T}{\partial q_{\mathrm{f}, 0}^{\prime \prime}}}_{=0}+\widetilde{T} \frac{\partial}{\partial q_{\mathrm{f}, 0}^{\prime \prime}}\left(\frac{q_{\mathrm{f}, 0}^{\prime \prime} L}{k}\right)] \\
& =\frac{q_{\mathrm{f}, 0}^{\prime \prime} L}{k} \widetilde{T}
\end{aligned}
$$

which in dimensionless form results in $\widetilde{X}_{q_{\mathrm{f}, 0}^{\prime \prime}}=\widetilde{T}$, where $\widetilde{T}$ is defined by Eq. (11a). In the same way it is found that $\widetilde{X}_{q_{\mathrm{f}, 0}^{\prime \prime}, \mathrm{f}}=$ $\widetilde{T}_{\mathrm{f}}$ where now $\widetilde{T}_{\mathrm{f}}$ is defined by Eq. (11c). Therefore, the curves shown in Figure 2 also represent the sensitivity coefficient for the finite body to the applied heat flux.

4.2. Thermal Conductivity and Volumetric Heat Capacities as Parameters. As $\widetilde{T}$ and $\widetilde{T}_{\mathrm{f}}$ temperatures depend on the thermal conductivity of the specimen $k$ and volumetric heat capacity of both specimen and heater, $C$ and $C_{f}$, respectively, six sensitivity coefficients should be studied.
When the considered parameter is the thermal conductivity, the corresponding sensitivity coefficient is given as

$$
\begin{aligned}
X_{k} & =k \frac{\partial T}{\partial k}=k \frac{\partial}{\partial k}\left(\widetilde{T} \frac{q_{\mathrm{f}, 0}^{\prime \prime} L}{k}\right) \\
& =k\left[\frac{q_{\mathrm{f}, 0}^{\prime \prime} L}{k} \frac{\partial \widetilde{T}}{\partial k}+\widetilde{T} \frac{\partial}{\partial k}\left(\frac{q_{\mathrm{f}, 0}^{\prime \prime} L}{k}\right)\right] \\
& =k\left[\frac{q_{\mathrm{f}, 0}^{\prime \prime} L}{k} \frac{\partial \widetilde{T}}{\partial k}-\frac{q_{\mathrm{f}, 0}^{\prime \prime} L}{k^{2}} \widetilde{T}\right]
\end{aligned}
$$

where $\widetilde{T}=\widetilde{T}\left[\widetilde{t}, P, \widetilde{R}_{\mathrm{c}}, \beta_{m}\left(P, \widetilde{R}_{\mathrm{c}}\right)\right]$, with $\widetilde{t}[\alpha(k, C)], P\left(C, C_{\mathrm{f}}\right)$, $\widetilde{R}_{\mathrm{c}}\left(R_{\mathrm{c}}, k\right)$. Thus, by using the chain rule,

$$
\frac{\partial \widetilde{T}}{\partial k}=\frac{\partial \widetilde{T}}{\partial \widetilde{t}} \frac{\partial \tilde{t}}{\partial \alpha} \frac{\partial \alpha}{\partial k}+\frac{\partial \widetilde{T}}{\partial \widetilde{R}_{\mathrm{c}}} \frac{\partial \widetilde{R}_{\mathrm{c}}}{\partial k}+\sum_{m=1}^{\infty} \frac{\partial \widetilde{T}}{\partial \beta_{m}} \frac{\partial \beta_{m}}{\partial \widetilde{R}_{\mathrm{c}}} \frac{\partial \widetilde{R}_{\mathrm{c}}}{\partial k}
$$

Substituting Eq. (21) in Eq. (20) gives the scaled sensitivity coefficient with respect to the thermal conductivity. In dimensionless form it becomes

$$
\begin{aligned}
\widetilde{X}_{k} & =k \frac{\partial \widetilde{T}}{\partial k}-\widetilde{T} \\
& =\widetilde{t} \frac{\partial \widetilde{T}}{\partial \widetilde{t}}+\widetilde{R}_{\mathrm{c}}\left[\frac{\partial \widetilde{T}}{\partial \widetilde{R}_{\mathrm{c}}}+\sum_{m=1}^{\infty} \frac{\partial \widetilde{T}}{\partial \beta_{m}} \frac{\partial \beta_{m}}{\partial \widetilde{R}_{\mathrm{c}}}\right]-\widetilde{T}
\end{aligned}
$$

In the same way, the sensitivity coefficient with respect to the volumetric heat capacity $C$ may be obtained.

$$
\widetilde{X}_{C}=C \frac{\partial \widetilde{T}}{\partial C}=-\tilde{t} \frac{\partial \widetilde{T}}{\partial \tilde{t}}-P \frac{\partial \widetilde{T}}{\partial P}-P \sum_{m=1}^{\infty} \frac{\partial \widetilde{T}}{\partial \beta_{m}} \frac{\partial \beta_{m}}{\partial P}
$$


Similarly the sensitivity coefficient with respect to the volumetric heat capacity of the heater $C_{f}$, in dimensionless form, results in

$$
\widetilde{X}_{C_{\mathrm{f}}}=C_{\mathrm{f}} \frac{\partial \widetilde{T}}{\partial C_{\mathrm{f}}}=P \frac{\partial \widetilde{T}}{\partial P}+P \sum_{m=1}^{\infty} \frac{\partial \widetilde{T}}{\partial \beta_{m}} \frac{\partial \beta_{m}}{\partial P}
$$

As regards the thin layer sensitivities, they are defined as

$$
\begin{aligned}
\widetilde{X}_{k, \mathrm{f}} & =k \frac{\partial \widetilde{T}_{\mathrm{f}}}{\partial k}-\widetilde{T}_{\mathrm{f}} \\
& =\widetilde{t} \frac{\partial \widetilde{T}_{\mathrm{f}}}{\partial \widetilde{t}}+\widetilde{R}_{\mathrm{c}}\left[\frac{\partial \widetilde{T}_{\mathrm{f}}}{\partial \widetilde{R}_{\mathrm{c}}}+\sum_{m=1}^{\infty} \frac{\partial \widetilde{T}_{\mathrm{f}}}{\partial \beta_{m}} \frac{\partial \beta_{m}}{\partial \widetilde{R}_{\mathrm{c}}}\right]-\widetilde{T}_{\mathrm{f}} \\
\widetilde{X}_{C, \mathrm{f}} & =C \frac{\partial \widetilde{T}_{\mathrm{f}}}{\partial C}=-\widetilde{t} \frac{\partial \widetilde{T}_{\mathrm{f}}}{\partial \tilde{t}}-P \frac{\partial \widetilde{T}_{\mathrm{f}}}{\partial P}-P \sum_{m=1}^{\infty} \frac{\partial \widetilde{T}_{\mathrm{f}}}{\partial \beta_{m}} \frac{\partial \beta_{m}}{\partial P} \\
\widetilde{X}_{C_{\mathrm{f}}, \mathrm{f}} & =C \widetilde{f}_{\mathrm{f}} \frac{\partial \widetilde{T}_{\mathrm{f}}}{\partial C_{\mathrm{f}}}=P \frac{\partial \widetilde{T}_{\mathrm{f}}}{\partial P}+P \sum_{m=1}^{\infty} \frac{\partial \widetilde{T}_{\mathrm{f}}}{\partial \beta_{m}} \frac{\partial \beta_{m}}{\partial P}
\end{aligned}
$$

4.3. Contact Resistance as a Parameter. If the parameter of interest is the thermal contact resistance $R_{\mathrm{c}}$, the corresponding sensitivity coefficients are

$$
\begin{gathered}
\widetilde{X}_{R_{\mathrm{c}}}=R_{\mathrm{C}} \frac{\partial \widetilde{T}}{\partial R_{\mathrm{C}}}=\widetilde{R}_{\mathrm{c}} \frac{\partial \widetilde{T}}{\partial \widetilde{R}_{\mathrm{c}}}+\widetilde{R}_{\mathrm{c}} \sum_{m=1}^{\infty} \frac{\partial \widetilde{T}}{\partial \beta_{m}} \frac{\partial \beta_{m}}{\partial \widetilde{R}_{\mathrm{c}}} \\
\widetilde{X}_{R_{\mathrm{c}}, \mathrm{f}}=R_{\mathrm{c}} \frac{\partial \widetilde{T}_{\mathrm{f}}}{\partial R_{\mathrm{c}}}=\widetilde{R}_{\mathrm{c}} \frac{\partial \widetilde{T}_{\mathrm{f}}}{\partial \widetilde{R}_{\mathrm{c}}}+\widetilde{R}_{\mathrm{c}} \sum_{m=1}^{\infty} \frac{\partial \widetilde{T}_{\mathrm{f}}}{\partial \beta_{m}} \frac{\partial \beta_{m}}{\partial \widetilde{R}_{\mathrm{c}}}
\end{gathered}
$$

It is interesting to note that all scaled sensitivity coefficients discussed above are linearly dependent; in fact, they sum to zero for all values of time and position; i.e.,

$$
\begin{gathered}
\left(\widetilde{X}_{q_{\mathrm{f}, 0}^{\prime \prime}}+\widetilde{X}_{q_{\mathrm{f}, 0}^{\prime \prime} \mathrm{f}}\right)+\left(\widetilde{X}_{k}+\widetilde{X}_{k, \mathrm{f}}\right)+\left(\widetilde{X}_{C}+\widetilde{X}_{C, \mathrm{f}}\right) \\
+\left(\widetilde{X}_{C_{\mathrm{f}}}+\widetilde{X}_{C_{\mathrm{f}}, \mathrm{f}}\right)-\left(\widetilde{X}_{R_{\mathrm{c}}}+\widetilde{X}_{R_{\mathrm{c}} \mathrm{f}}\right)=0
\end{gathered}
$$

The same condition is valid for the thin layer (heater) and the finite body (sample) considered individually:

$$
\begin{array}{r}
\widetilde{X}_{k, \mathrm{f}}+\widetilde{X}_{C, \mathrm{f}}+\widetilde{X}_{C_{\mathrm{f}}, \mathrm{f}}-\widetilde{X}_{R_{\mathrm{c}} \mathrm{f}}=-\widetilde{T}_{\mathrm{f}} \\
\widetilde{X}_{k}+\widetilde{X}_{C}+\widetilde{X}_{C_{\mathrm{f}}}-\widetilde{X}_{R_{c}}=-\widetilde{T}
\end{array}
$$

4.4. Computation of the Scaled Sensitivity Coefficients. The sensitivity coefficients listed above may be computed analytically. In fact, the temperature derivatives with respect to $\tilde{t}$, $P$, and $\widetilde{R}_{\mathrm{c}}$ can be taken directly using Eqs. (11a) and (11c). As regards the derivatives $\partial \beta_{m} / \partial P$ and $\partial \beta_{m} / \partial \widetilde{R}_{c}$, from the eigencondition defined by Eq. (12) one can obtain

$$
\begin{aligned}
P & =\frac{\tan \left(\beta_{m}\right)}{\beta_{m}^{2} \widetilde{R}_{\mathrm{c}} \tan \left(\beta_{m}\right)-\beta_{m}}, \\
\widetilde{R}_{\mathrm{c}} & =\frac{\beta_{m}+\tan \left(\beta_{m}\right) / P}{\beta_{m}^{2} \tan \left(\beta_{m}\right)}
\end{aligned}
$$

Then, by differentiating Eq. (30) with respect to the eigenvalue $\beta_{m}$ and by taking the corresponding reciprocals, it is found that

$$
\begin{aligned}
& \frac{\partial \beta_{m}}{\partial P}=\frac{\left[\beta_{m}^{2} \widetilde{R}_{\mathrm{c}} \tan \left(\beta_{m}\right)-\beta_{m}\right]^{2}}{\tan \left(\beta_{m}\right)\left[1-\beta_{m} \tan \left(\beta_{m}\right)\left(1+2 \widetilde{R}_{\mathrm{c}}\right)\right]-\beta_{m}}, \\
& \frac{\partial \beta_{m}}{\partial \widetilde{R}_{\mathrm{c}}}=-\frac{\beta_{m}^{2} \tan \left(\beta_{m}\right)}{1+\tan \left(\beta_{m}\right)\left(2 / P \beta_{m}+\beta_{m}\right)}
\end{aligned}
$$

In addition, for early times (less than the deviation time $\left.\tilde{t}_{\mathrm{d}}\right)$ the temperature field within the specimen and the thin layer temperature may be evaluated accurately by using the semi-infinite transient solution defined in [19].

4.5. Sensitivity Analysis Results. Three different locations are considered in the analysis: the interface between the heater and the sample, i.e., $\widetilde{x}=0$ (both heater side and sample side) and the sample backside $(\tilde{x}=1)$. Note that two sensors are used at the interface $\tilde{x}=0$, one located on the surface of the heater and the other on the surface of the sample. Then at this interface a silicon grease is used to reduce the contact resistance. Note also that the sensor location on the surface of the heater (interface, heater side) is realistic only when using kapton or silicon resistive heaters rather than metallic ones.

Figures 3-5 show the sample temperature sensitivities as a function of time and for different values of the heat capacity ratio $P$; also, the thin heater sensitivities $\widetilde{X}_{C, \mathrm{f}}$ and $\widetilde{X}_{C_{f}, \mathrm{f}}$ are shown by Figure 6 . For the first group of figures a curve for $P=0$ is plotted as a reference case; in fact, in such a case, the thin heater is neglected and the analyzed problem reduces to the X22B10T0 case whose solution is discussed in [21].

Figure 3 shows the sensitivity coefficient $\widetilde{X}_{k}$. In particular, for $\tilde{x}=0$, it decreases in absolute value when the $P$ parameter increases, while at the backside $(\tilde{x}=1)$ this behaviour cannot be observed for $P=0.1$. Also, at $\tilde{x}=1$ the sensitivity to the thermal conductivity $k$ is less affected by the $P$ parameter than the sensitivity at $\tilde{x}=0$, when large times are considered.

The sensitivity coefficients $\widetilde{X}_{C}$ and $\widetilde{X}_{C_{f}}$ for $\tilde{x}=0$ are depicted in Figure 4. The corresponding graphs for $\tilde{x}=1$ are not reported here as they are similar to ones given in Figure 4. A comparison among Figures 4(a) and 4(b) suggests that, at $\tilde{x}=0$, the sample temperature is more sensitive to its heat capacity $C$ when $P<0.5$, while for $P \geq 0.5$ it becomes more sensitive to that of the thin heater $C_{f}$. Also, the sample temperature sensitivity to the contact resistance $R_{\mathrm{c}}$ is shown in Figure 5. As indicated by the figure, the highest sensitivity occurs at the interface between the heater and the sample $(\tilde{x}=0)$.

The coefficients $\widetilde{X}_{C, \mathrm{f}}$ and $\widetilde{X}_{C_{\mathrm{f}}, \mathrm{f}}$ related to the thin heater are shown by Figure 6 . It is worth noting that, at large times and for $P<0.5$, the heater temperature is more sensitive to the volumetric heat capacity of the sample $C$ than to its volumetric heat capacity $C_{\mathrm{f}}$; on the contrary, for $P \geq 0.5$ it becomes more sensitive to $C_{\mathrm{f}}$.

Figures 7 and 8 show the sensitivities $\widetilde{X}_{k}($ at $\tilde{x}=0)$ and $\widetilde{X}_{k, \mathrm{f}}$ and the other heater coefficients $\widetilde{X}_{C_{\mathrm{f}}, \mathrm{f}}$ and $\widetilde{X}_{R_{\mathrm{c}}, \mathrm{f}}$, respectively, as a function of time with the dimensionless 


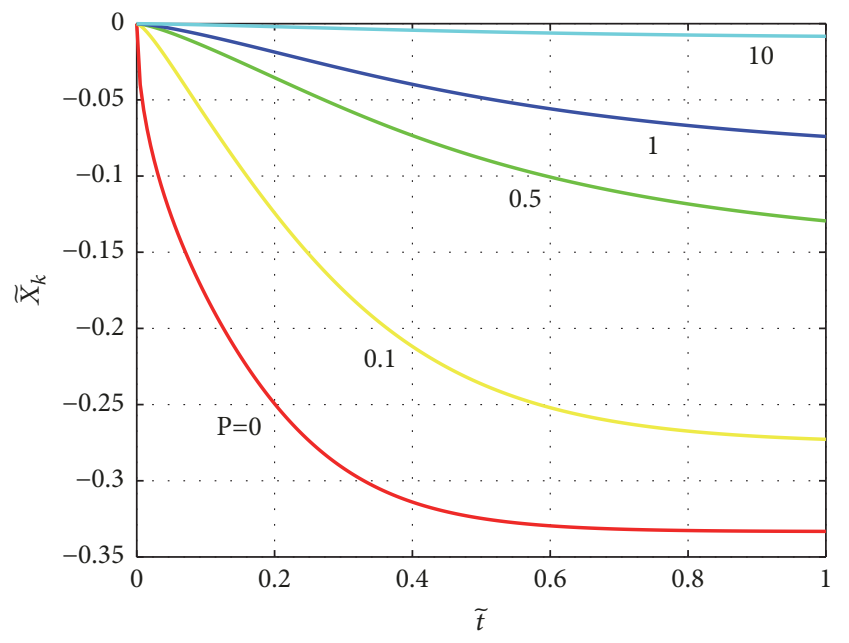

(a)

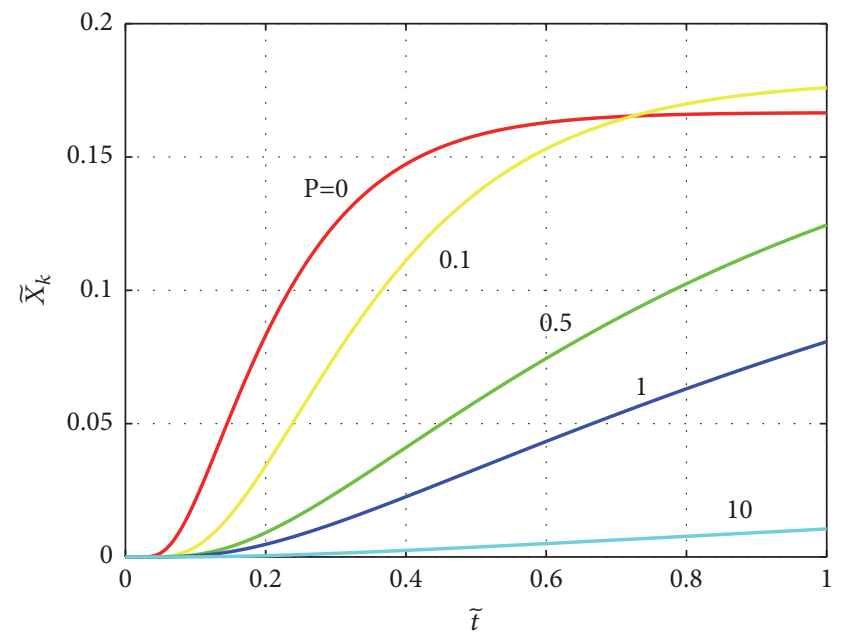

(b)

FIGURE 3: Sensitivity coefficient to the thermal conductivity as a function of time, for different $P$ values and $\widetilde{R}_{\mathrm{c}}=1$, at $\tilde{x}=0$ (a) and at $\tilde{x}=1$ (b).

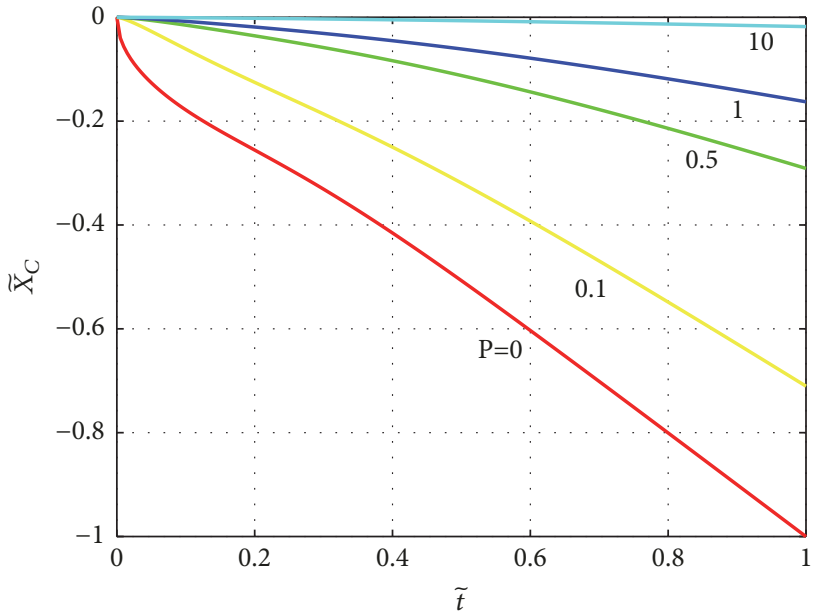

(a)

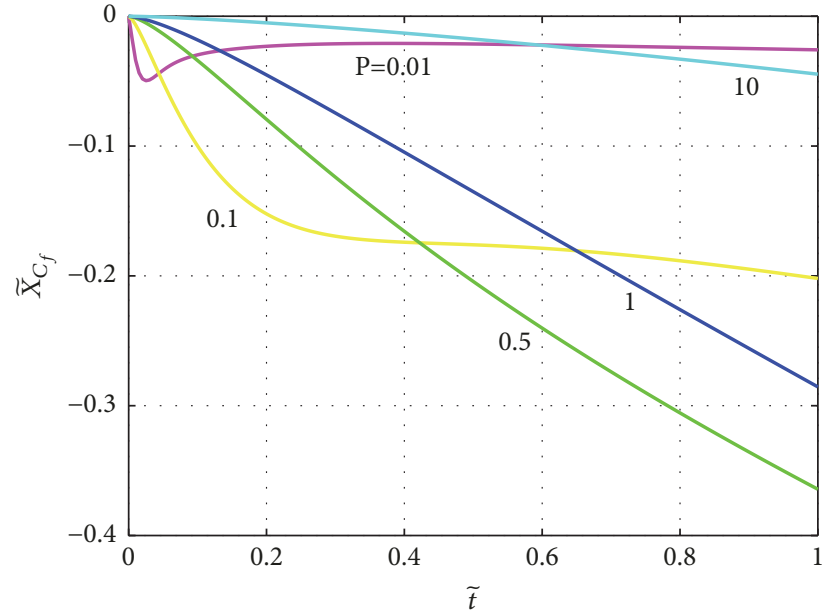

(b)

FIGURE 4: Sensitivity coefficients to the sample heat capacity (a) and to the heater heat capacity (b) as a function of time, for different $P$ values and $\widetilde{R}_{\mathrm{c}}=1$, at $\widetilde{x}=0$.

contact resistance $\widetilde{R}_{\mathrm{c}}$ as a parameter. In these figures a curve for $\widetilde{R}_{c}=0$ is plotted as a reference case; in fact, in such a case, the contact between the heater and the sample is perfect and the problem reduces to the X42B10T00 case, whose solution is provided in [10] and discussed in [22]. Also, a sensitivity analysis for this heat conduction problem is carried out in [23]. Note that the heater sensitivities are more affected by the thermal contact resistance $\widetilde{R}_{c}$, than the sample sensitivity coefficients.

A comparison of the sensitivity coefficients related to the sample and to the heater at $\tilde{x}=0$ and for $P=\widetilde{R}_{\mathrm{c}}=0.1$ is given by Figure 9. In particular, Figure 9 (a) compares the coefficients $\widetilde{X}_{k}, \widetilde{X}_{C}, \widetilde{X}_{k, \mathrm{f}}$ and $\widetilde{X}_{C, \mathrm{f}}$, while Figure 9 (b) compares the coefficients $\widetilde{X}_{C_{\mathrm{f}}}, \widetilde{X}_{R_{c}}, \widetilde{X}_{C_{\mathrm{f}}, \mathrm{f}}$, and $\widetilde{X}_{R_{\mathrm{c}}, \mathrm{f}}$. The former shows that, for times less than the deviation time defined by Eq. (16), the coefficients $\widetilde{X}_{k}$ and $\widetilde{X}_{C}$ are exactly equal for $\tilde{x}=0$, and the same equality is also true for $\widetilde{X}_{k, \mathrm{f}}$ and $\widetilde{X}_{C, \mathrm{f}}$. Moreover, the sensitivities to the thermal conductivity $k$, i.e., $\widetilde{X}_{k}$ and $\widetilde{X}_{k, \mathrm{f}}$, respond in the same way; it also occurs for the coefficients $\widetilde{X}_{C}$ and $\widetilde{X}_{C, \mathrm{f}}$. This suggests that for small values of $P$ and $\widetilde{R}_{\mathrm{c}}$, it is indifferent to place the temperature sensor on the heater side or on the sample side. In addition, for large values of these parameters, the sensitivities to $k$ and $C$ at the sample side are greater than the corresponding heater coefficients (i.e., $\widetilde{X}_{k, \mathrm{f}}$ and $\widetilde{X}_{C, \mathrm{f}}$ ). On the contrary, Figure 9 (b) shows that the greatest sensitivities to the heater heat capacity $C_{\mathrm{f}}$ and to the thermal contact resistance $R_{\mathrm{c}}$ occur at the heater side. This consideration is always valid for the sensitivity to $C_{\mathrm{f}}$ regardless of the value of $P$ and $\widetilde{R}_{\mathrm{c}}$; as regards the sensitivity to the contact resistance it is valid only for $P \leq 0.5$. 


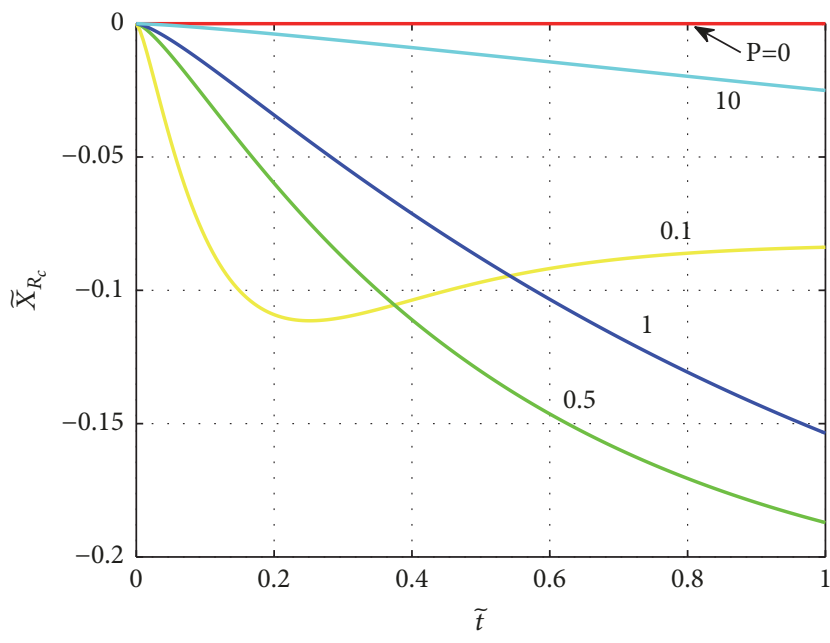

(a)

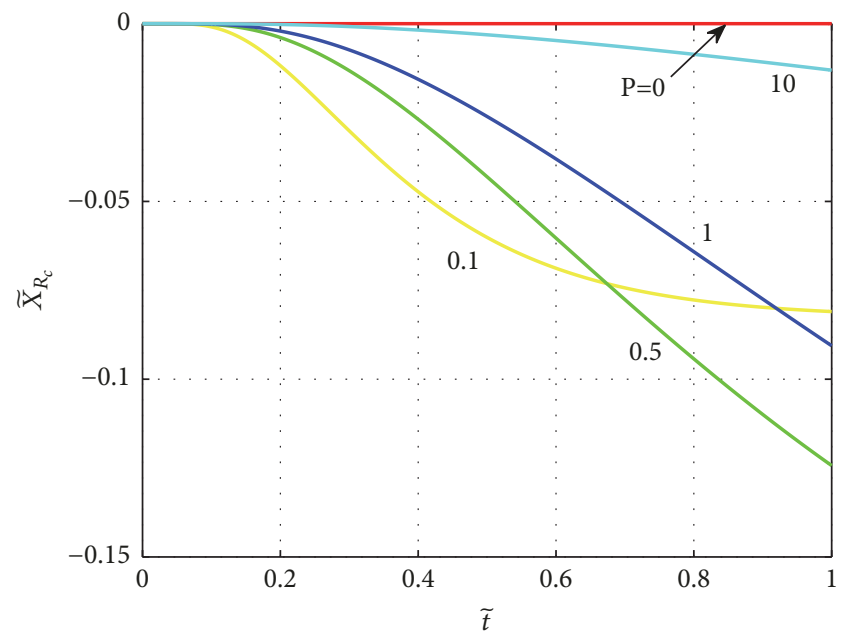

(b)

FIGURE 5: Sensitivity coefficient to the thermal contact resistance as a function of time, for different $P$ values and $\widetilde{R}_{\mathrm{c}}=1$, at $\widetilde{x}=0$ (a) and at $\tilde{x}=1(b)$.

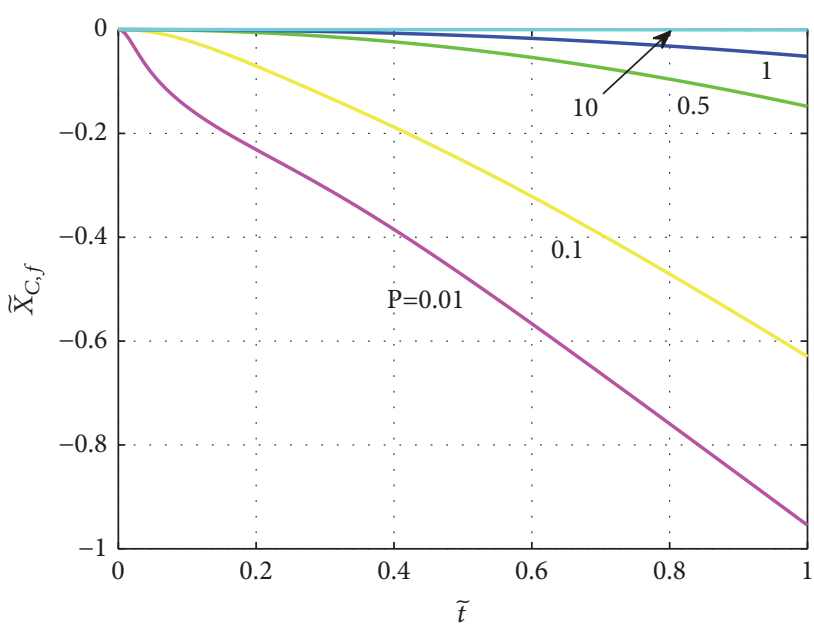

(a)

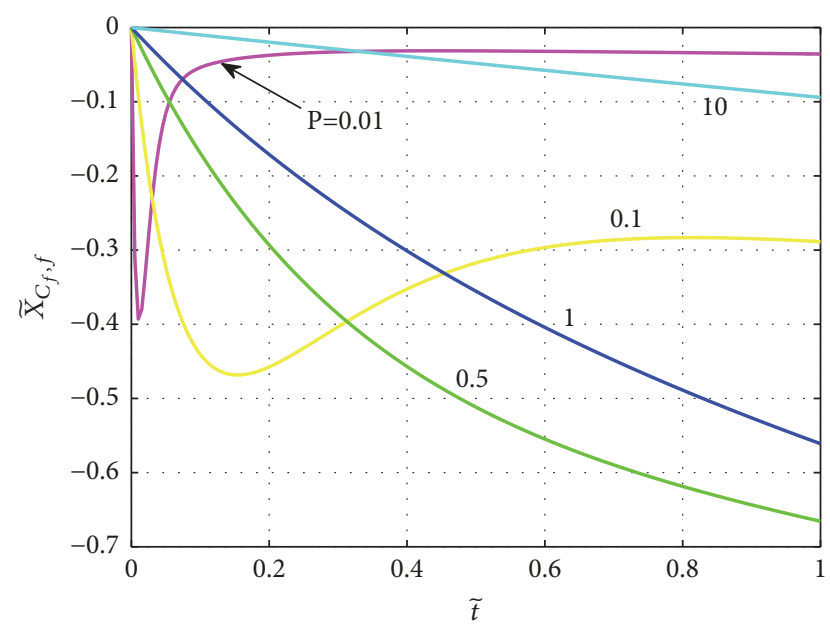

(b)

Figure 6: Heater sensitivity to the sample heat capacity (a) and to its heat capacity (b) as a function of time, for different $P$ values and $\widetilde{R}_{\mathrm{c}}=1$.

Figure 10 compares the sample sensitivities with respect to $k, C, C_{\mathrm{f}}$, and $R_{\mathrm{c}}$ obtained for $P=\widetilde{R}_{\mathrm{c}}=0.1$, at the sample backside $(\tilde{X}=1)$. As shown by the figure the coefficients $\widetilde{X}_{k}, \widetilde{X}_{C}, \widetilde{X}_{C_{\mathrm{f}}}$, and $\widetilde{X}_{R_{c}}$ respond initially in the same way, and then they split up and in particular the sensitivity to $k$ approaches to a constant value, while the sensitivity to $C$ increases linearly with the time. Therefore, at the sample backside the coefficients $\widetilde{X}_{k}$ and $\widetilde{X}_{C}$ are uncorrelated, and the thermal properties $k$ and $C$ can be estimated simultaneously. Furthermore, as no large magnitude difference exists between them the estimates for $k$ and $C$ may be accurate.

Also, by comparing Figures 9(a) and 10, it is possible to state that the greatest sensitivity to the sample heat capacity $C$ always occurs at the backside $\widetilde{x}=1$ (except at early times). As far as the sensitivity to $k$ is concerned, for small values of $P$ (around 0.1$)$ it is greater at the sample side $(\tilde{x}=0$ ), while, for large $P$ values and at large times, it becomes greater at the sample backside. Also, the analysis shows that for estimating the thermal conductivity $k$, in the event that the heat capacity ratio $(P)$ is unknown, the temperature sensor should be placed at $\tilde{x}=1$, where the influence of this parameter is lower (see Figure 3).

Moreover, at the sample backside the sensitivities to the heater heat capacity $C_{\mathrm{f}}$ and to the contact resistance $R_{\mathrm{c}}$ are lower than the corresponding coefficients obtained for $\tilde{x}=0$. In particular, for estimating $C_{\mathrm{f}}$ the temperature sensor should be placed at the heater side when a thin enough heater $(P \leq$ $0.5)$ is used and large times are considered. In fact, at large times the influence of $R_{c}$ is the same either at the heater side or at the sample side, but at the heater side the sensitivity to $C_{\mathrm{f}}$ is higher. Also, it is even the best location for the temperature 


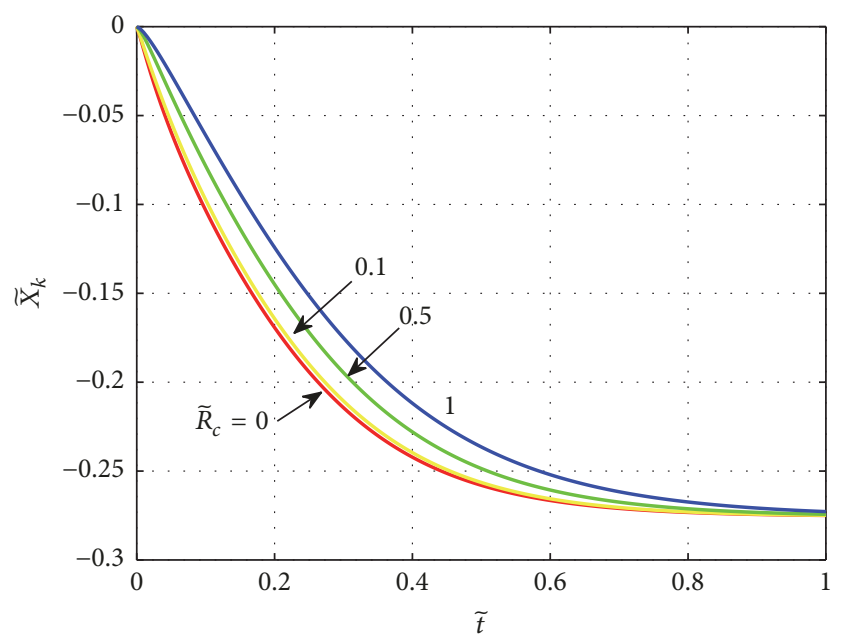

(a)

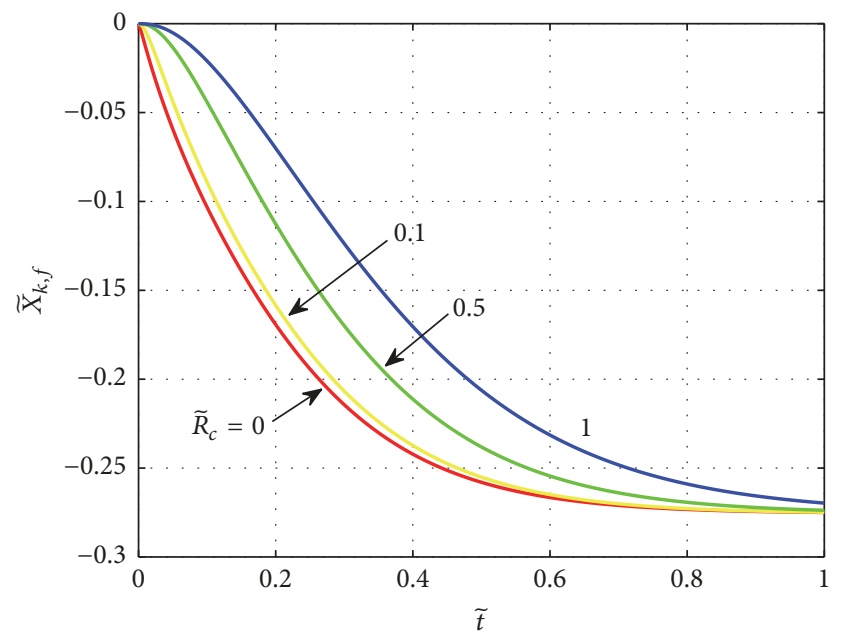

(b)

FIgURE 7: Sensitivity coefficients to the thermal conductivity as a function of time, for different $\widetilde{R}_{\mathrm{c}}$ values and $P=0.1$, for the sample at $\widetilde{x}=0$ (a) and for the heater (b).

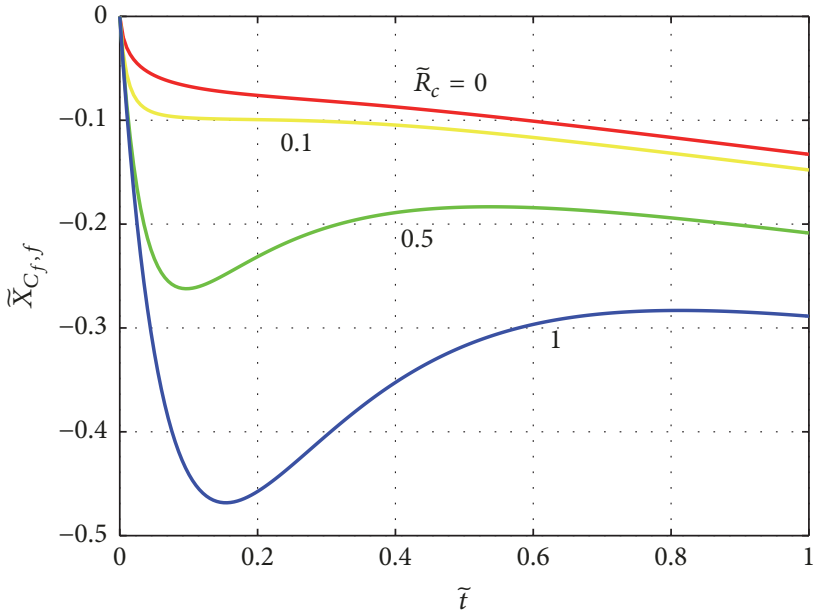

(a)

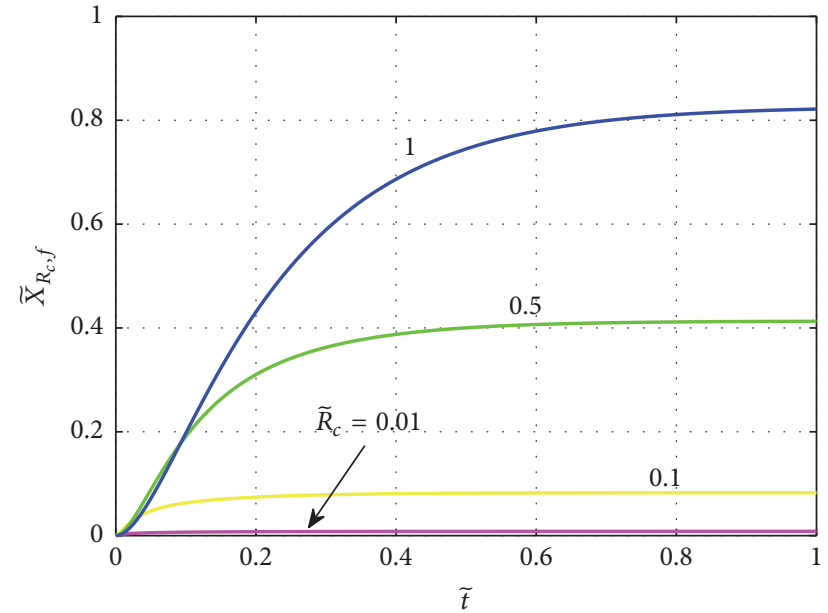

(b)

FiguRE 8: Sensitivity coefficients for the heater as a function of time, for different $\widetilde{R}_{\mathrm{c}}$ values and $P=0.1$, with respect to its heat capacity (a) and to the contact resistance (b).

sensor when estimating the contact resistance, in the event that a heater with known properties is used.

\section{Experimental Results}

The proposed methodology is now applied to a lab-controlled experiment based on the schematic of Figure 1(a) whose measured results are available in Ref. [8, Chapter 7, p. 402]. In this experiment two adjacent, identical Armco iron disk-shaped flat specimens (thickness $L=2.54 \times 10^{-2} \mathrm{~m}$; diameter $7.62 \times 10^{-2} \mathrm{~m}$ ) were heated by a Kapton heater $\left(2 L_{f}=8.4 \times 10^{-4} \mathrm{~m} ; C_{f}=2000 \mathrm{~kJ} / \mathrm{m}^{3 \circ} \mathrm{C}\right)$ placed between them. Also, an imperfect contact between the heater and the sample is considered through a contact resistance $R_{c}=1.34 \times 10^{-4} \mathrm{~m}^{2 \circ} \mathrm{C} / \mathrm{W}$. All the external surfaces of the samples were insulated. Therefore, the heat conduction may be considered one dimensional. Moreover four temperatures sensors were carefully attached to the heated surface of each specimen and four were attached at the insulated backsides. In particular, the sensors were located at angles of $0^{\circ}, 90^{\circ}$, $180^{\circ}$, and $270^{\circ}$.

Both specimens were at the same uniform initial temperature (about $27.5^{\circ} \mathrm{C}$ ) before the start of the experiment. Though the heat flux of $30.3 \mathrm{~kW} / \mathrm{m}^{2}$ was applied to each sample for a finite heating duration of $15.3 \mathrm{~s}$, the measured data were collected for $27 \mathrm{~s}$ with a time step of $0.3 \mathrm{~s}$.

The average measured temperatures at both the heated $(x=0)$ and insulated $(x=L)$ surfaces are shown in Figure 11(a). It is evident that the heated surface temperature increases only during the heating period, while the insulated surface 


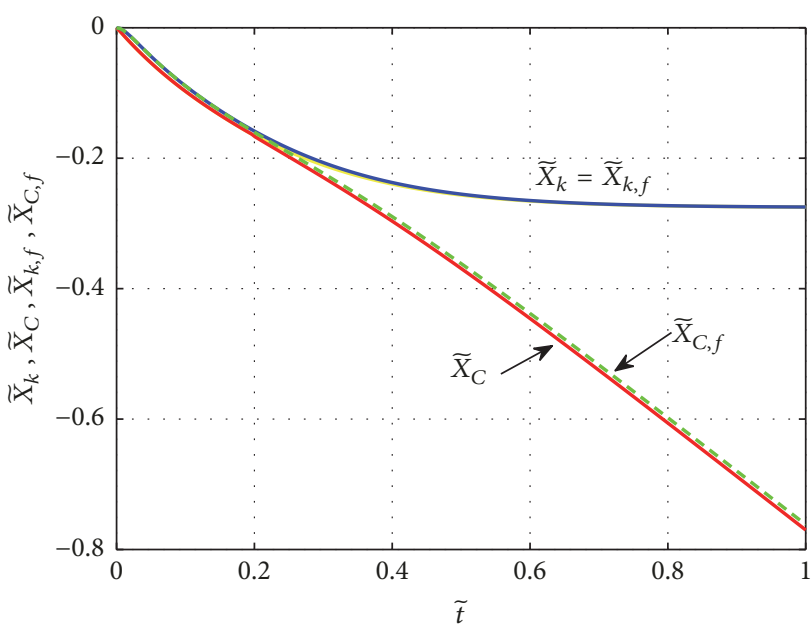

(a)

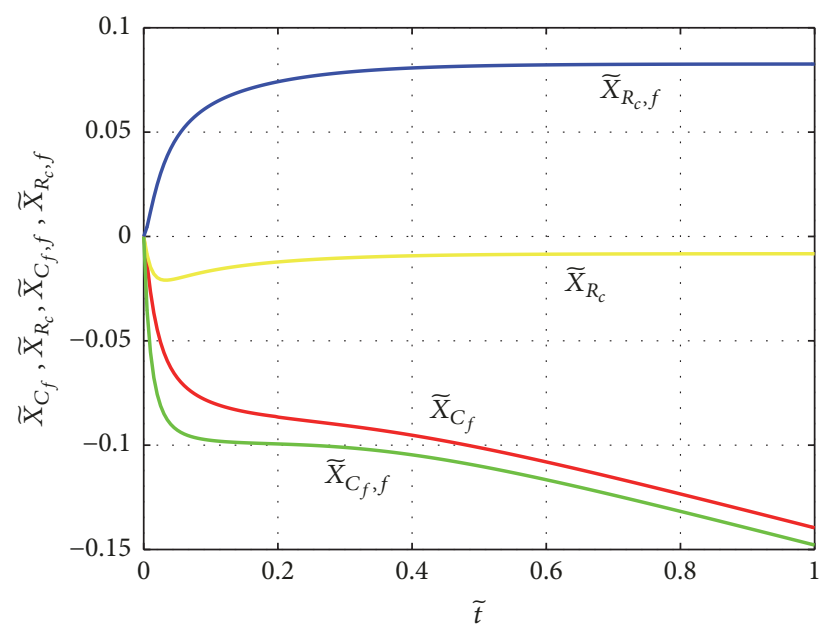

(b)

Figure 9: Comparison of the sensitivity coefficients of the heater and of the sample at $\tilde{x}=0$, with respect to the thermal conductivity and to the sample heat capacity (a), and with respect to the heater heat capacity and to the contact resistance (b) for $P=\widetilde{R}_{\mathrm{c}}=0.1$.

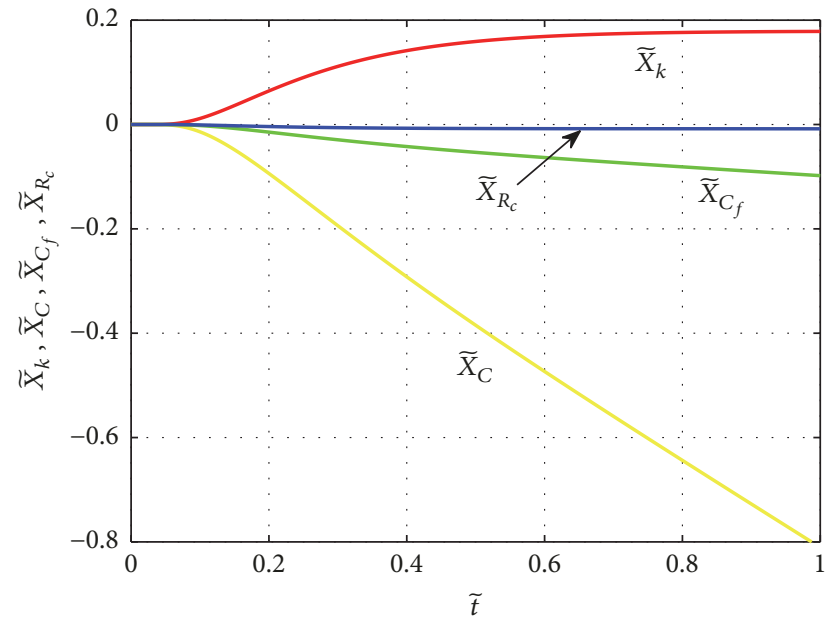

FIGURE 10: Comparison of the sensitivity coefficients related to the sample, at $\tilde{x}=1$ and for $P=\widetilde{R}_{\mathrm{c}}=0.1$.

temperature continues to increase after heater is turned off until they approach the same equilibrium temperature. Figures 11(b) and 11(c) show the dimensional actual sensitivity coefficients with respect to $k$ and $C$, respectively, for the X22B50T0 (heater completely neglected $\Longrightarrow C_{f}=0$ and $R_{c}=0$ ) and X62B50T00 cases, where, in the numbering system [13, Table 2], "B5" denotes a step change in time for a finite heating duration. It can be observed that the sensitivity coefficients are not very different at the same location as the heater used is very thin and the contact resistance is very low (in fact, it was reduced using a silicon grease).

Sensitivity coefficients are involved in the parameter estimation procedure when minimizing the ordinary least squares norm

$$
S=\sum_{s=1}^{8} \sum_{n=1}^{90}\left[Y_{s}(n)-T_{s}(n)\right]^{2}
$$

where $Y_{s}(n)$ is the measured temperature from the $s$-th sensor at $n$-th time and $T_{s}(n)$ is the calculated temperature at the location of the $s$-th sensor at $n$-th time. Then, applying the Gauss method to minimize Eq. (32) yields the following recursive expression:

$$
\underline{b}^{(i+1)}=\underline{b}^{(i)}+\left[\underline{X}^{T(i)} \underline{X}^{(i)}\right]^{-1} \underline{X}^{T(i)}\left[\underline{Y}-\underline{T}^{(i)}\right]
$$

where $i$ is the iteration index, $\underline{b}$ denotes the estimated parameter vector (in the current case $\underline{b}=\left[\begin{array}{ll}k & C\end{array}\right]^{T}$ ), $\underline{Y}$ is the measured temperature vector, $\underline{T}$ is the calculated temperature vector, and $X$ is the sensitivity coefficient matrix. Also, in Eq. (33) $\underline{X}^{(i)}, \underline{X}^{T(i)}$, and $\underline{T}^{(i)}$ are all computed using the $\underline{b}^{(i)}$ vector obtained at the $i$-th iteration.

Moreover, the uncertainty related to the estimated parameters $(k$ and $C$ ) is determined as discussed in the next subsection.

5.1. Uncertainty Quantification. Under standard statistical assumptions of additive, uncorrelated, normal errors with zero mean and constant variance the approximate covariance matrix of estimated parameters can be written as $[8$, p. 452 , Eq. 8.5.17]

$$
\begin{aligned}
\operatorname{cov}(\underline{\mathrm{b}}) & =\left[\begin{array}{cc}
\sigma_{k}^{2} & \sigma_{k, C} \\
\sigma_{k, C} & \sigma_{C}^{2}
\end{array}\right]=\left[\begin{array}{ll}
C_{k k} & C_{k C} \\
C_{k C} & C_{C C}
\end{array}\right]^{-1} \sigma^{2} \\
& =\left[\begin{array}{cc}
C_{C C} & -C_{k C} \\
-C_{k C} & C_{k k}
\end{array}\right] \frac{\sigma^{2}}{\Delta}
\end{aligned}
$$

where $\sigma^{2}$ is the estimated variance of measurement errors, $\Delta=C_{k k} C_{C C}-C_{k C}^{2}$ denotes the determinant of the $\underline{X}^{T} \underline{X}$ 


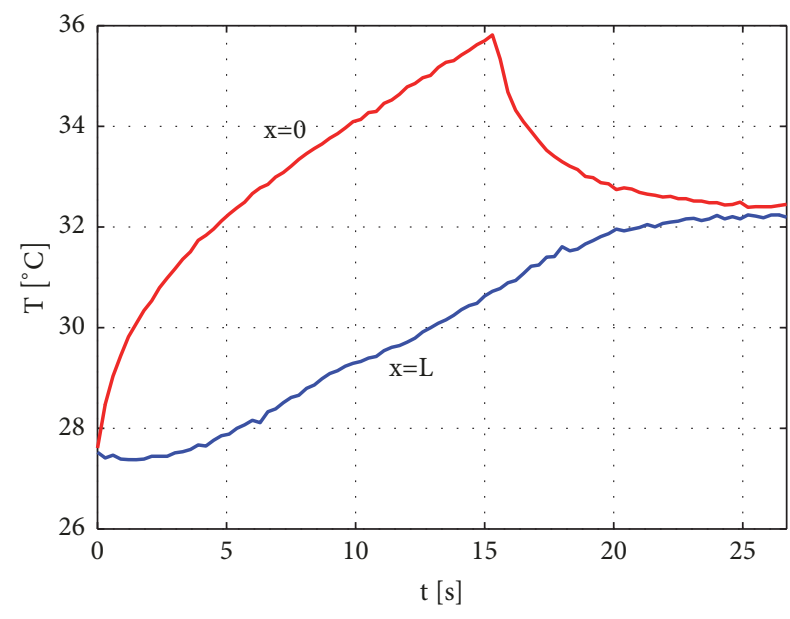

(a)

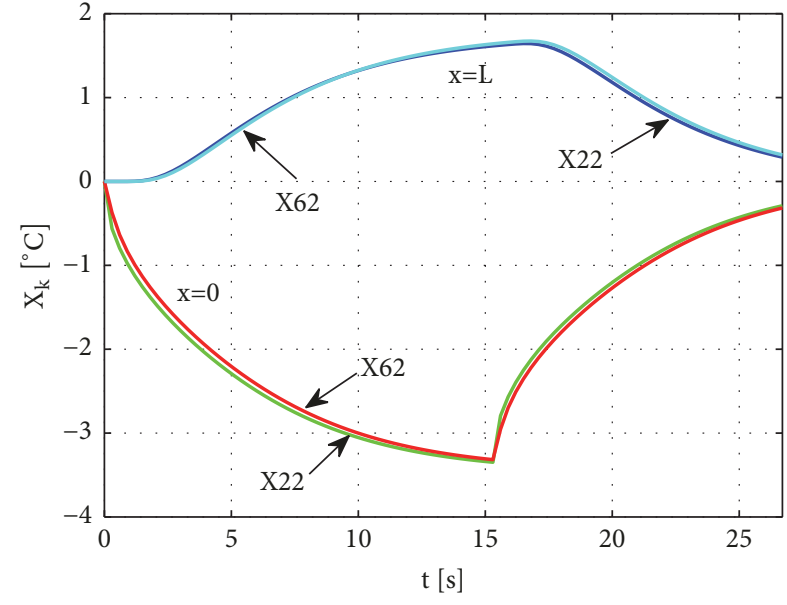

(b)

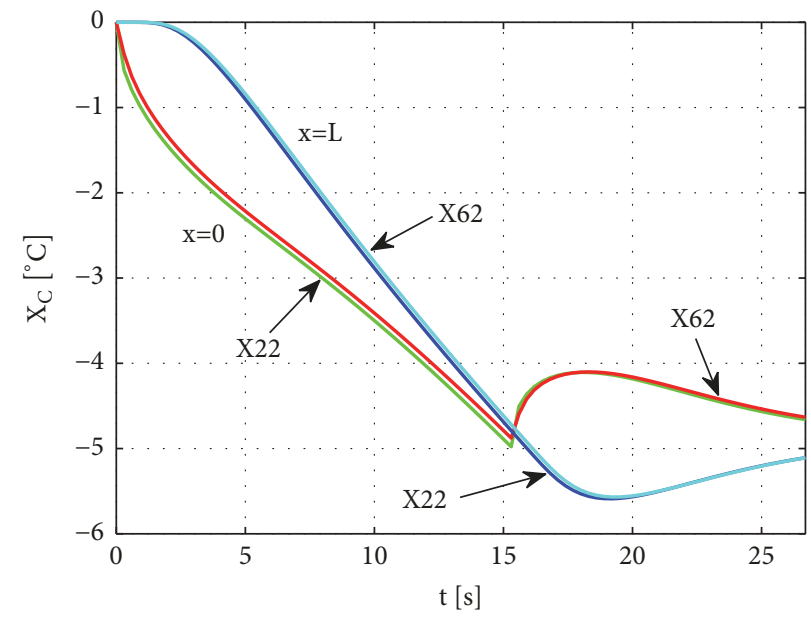

(c)

FIGURE 11: Average measured temperatures at $x=0$ and $x=L$ (a), dimensional actual sensitivity coefficients for X22B50T0 and X62B50T00 cases with respect to $k(\mathrm{~b})$, and with respect to $C(\mathrm{c})$.

matrix appearing in Eq. (33), and the elements $C_{k k}$, $C_{C C}$, and $C_{k C}$ are defined as

$$
\begin{aligned}
C_{k k} & =\sum_{s=1}^{8} \sum_{n=1}^{90}\left[\frac{\partial T_{s}(n)}{\partial k}\right]^{2} ; \\
C_{C C} & =\sum_{s=1}^{8} \sum_{n=1}^{90}\left[\frac{\partial T_{s}(n)}{\partial C}\right]^{2} ; \\
C_{k C} & =C_{C k}=\sum_{s=1}^{8} \sum_{n=1}^{90} \frac{\partial T_{s}(n)}{\partial k} \frac{\partial T_{s}(n)}{\partial C}
\end{aligned}
$$

Also, $\partial T_{s}(n) / \partial k$ and $\partial T_{s}(n) / \partial C$ (with $s=1, \ldots, 8$ ) are the sensitivity coefficients of temperature measured by the $s$ th sensor with respect to the thermal properties $k$ and $C$, respectively, at the $n$-th time.
Therefore, the standard deviations of $k$ and $C$ can be obtained from Eq. (34a) and (34b) as shown below.

$$
\begin{gathered}
\sigma_{k} \cong \sigma \sqrt{\frac{C_{C C}}{\Delta}} \\
\sigma_{C} \cong \sigma \sqrt{\frac{C_{k k}}{\Delta}}
\end{gathered}
$$

Once the standard deviations for $k$ and $C$ are known, a $90 \%$ confidence interval of the estimates can be computed.

The estimation procedure discussed above is applied to three different cases: (1) heater completely neglected (X22B50T0), (2) volumetric heater heat capacity and perfect thermal contact between the specimen and the heater (X42B50T00), and (3) volumetric heater heat capacity and imperfect contact between specimen and heater (X62B50T00). The results are shown in Table 2 where a comparison between the estimates of $k$ and $C$ obtained using the exact temperature solution (X22B50T0) and the finite difference method [8] is provided too. As in the current 
TABLE 2: Estimation procedure results for three different cases.

\begin{tabular}{lcccc}
\hline Case & $\mathrm{k}\left[\mathrm{W} /\left(\mathrm{m}^{\circ} \mathrm{C}\right)\right]$ & $\Delta \%$ & $\mathrm{C}\left[\mathrm{kJ} /\left(\mathrm{m}^{3 \circ} \mathrm{C}\right)\right]$ & \multicolumn{2}{c}{$\Delta \%$} \\
\hline X22B50T0 & $74.10 \pm 0.165$ & & $3736.95 \pm 3.707$ & 3728.60 \\
X22B50T0 [8] & 75.01 & 1.2 & $3692.35 \pm 3.691$ & -0.2 \\
X42B50T00 & $73.25 \pm 0.163$ & -1.2 & $3680.98 \pm 3.682$ & -1.2 \\
X62B50T00 & $73.16 \pm 0.163$ & -1.3 & -1.5 \\
\hline
\end{tabular}

experiment the standard deviations of the estimates are practically the same, the effect of heat source and contact resistance is of $-1.3 \%$ for the thermal conductivity and of $-1.5 \%$ for the volumetric heat capacity. However, the contact resistance between the heater and the specimen exhibits a lower influence. As already observed, this slight reduction is in accordance with the very thin heater and the very low contact resistance.

\section{Conclusions}

A novel mathematical modelling for simultaneous estimation of thermal properties of high-conductivity materials has been proposed. It allows the heater heat capacity and the surface contact resistance between sample and heater to be taken into account by using a nonconventional boundary condition, termed as a condition of the sixth kind.

The results of the analysis have been obtained by means of the Laplace transform method and the related residues theorem. They have shown that the heater heat capacity can affect the sample sensitivity coefficients more than the contact thermal resistance at their interface; and these coefficients are enough large and uncorrelated at the sample backside. Thus, this side was found to be the best location for the temperature sensor when estimating simultaneously thermal conductivity and volumetric heat capacity.

By comparing measured and calculated temperatures through the ordinary least squares norm and, then, minimizing it by the Gauss method, a reduction of about $1.4 \%$ was observed for the thermal properties values of Armco iron.

\section{Appendix}

\section{Residues Computation}

The calculation of the residues $R_{s=0}$ and $R_{s=-\beta_{m}^{2}}$ appearing in Eq. (9) is provided in the following.

In order to determine the residue at $q=0$ (or $s=0$ ), the inversion integrand $\vartheta(\widetilde{x}, q) e^{q^{2} \tilde{t}}$ (or $\vartheta(\widetilde{x}, s) e^{s \widetilde{t}}$ ) has to be brought into the form of a Laurent expansion at $q=0$; the sought residue corresponds to the coefficient of the $1 / q^{2}(1 / s)$ term appearing in the expansion.

By using a Taylor expansion for $e^{q^{2} t}$ and $\cosh [q(1-\tilde{x})]$ and bearing in mind Eq. (7), it is possible to note that $\vartheta(\widetilde{x}, q)$ is a quotient of even powers of $q$. Then, the inversion integrand can be rewritten as

$$
\begin{aligned}
\vartheta(\tilde{x}, q) e^{q^{2} \tilde{t}} \\
\quad=\frac{C_{0}+C_{1} q^{2}+O\left(q^{4}\right)}{q^{4}} \frac{1+\widetilde{t} q^{2}+O\left(q^{4}\right)}{C_{3}+C_{4} q^{2}+O\left(q^{4}\right)}
\end{aligned}
$$

which may be taken in a form closer to the Laurent expansion

$$
\begin{aligned}
\vartheta & (\widetilde{x}, q) e^{q \tilde{t}} \\
& =\left[\frac{C_{0}}{q^{4}}+\frac{C_{1}}{q^{2}}+O(1)\right]\left[C_{6}+C_{7} q^{2}+O\left(q^{4}\right)\right]
\end{aligned}
$$

Thus, bearing in mind Eq. (6) and by setting Eqs. (A.1a) and (A.1b) equal, one can obtain the following identities:

$$
\begin{aligned}
& C_{0}+C_{1} q^{2}+O\left(q^{4}\right)=\cosh [q(1-\tilde{x})] \\
& =1+\frac{q^{2}(1-\tilde{x})}{2}+O\left(q^{4}\right) \\
& q^{4}\left[C_{3}+C_{4} q^{2}+O\left(q^{4}\right)\right]=D(q) \\
& =q^{4}\left\{P+1+q^{2}\left[\frac{P}{2}+P \widetilde{R}_{c}+\frac{1}{6}\right]+O\left(q^{4}\right)\right\} \\
& \frac{1+\tilde{t} q^{2}+O\left(q^{4}\right)}{C_{3}+C_{4} q^{2}+O\left(q^{4}\right)}=C_{6}+C_{7} q^{2}+O\left(q^{4}\right)
\end{aligned}
$$

Then Eqs. (A.2a) and (A.2b) give

$$
\begin{aligned}
& C_{0}=1, \\
& C_{1}=\frac{(1-\widetilde{x})^{2}}{2}, \\
& C_{3}=P+1, \\
& C_{4}=\frac{P}{2}+P \widetilde{R}_{c}+\frac{1}{6}
\end{aligned}
$$

Also, Eq. (A.2c) is an identity and the constants $C_{6}$ and $C_{7}$ can be obtained similarly.

$$
\begin{aligned}
& C_{6}=\frac{1}{C_{3}}=\frac{1}{P+1}, \\
& C_{7}=\frac{1}{C_{3}}\left(\tilde{t}-\frac{C_{4}}{C_{3}}\right)
\end{aligned}
$$

Moreover, by using Eq. (A.1b) the coefficient of $1 / q^{2}(1 / s)$ term in the Laurent expansion, which is the residue $\left(R_{s=0}\right)$ for the pole $s=0$, may be identified.

$$
R_{s=0}=C_{0} C_{7}+C_{1} C_{6}
$$


Then by substituting Eqs. (A.3) and (A.4) into Eq. (A.5), after some algebra, the same expression reported in Eq. (10a) can be obtained.

Now consider the generic pole at $q=i \beta_{m}\left(\right.$ or $\left.s=-\beta_{m}^{2}\right)$. As first step, it is worth noting that the inversion integrand is a quotient of a numerator and a denominator, i.e., $\vartheta(\widetilde{x}, q) e^{q^{2} \widetilde{t}}=$ $N(q) / D(q)$; in such a case the residue for the simple pole $q=$ $i \beta_{m}$ may be conveniently calculated by taking the derivative of the denominator of Eq. (6) evaluated at its zero; that is,

$$
R_{s=-\beta_{m}^{2}}=\frac{N\left(q=i \beta_{m}\right)}{D^{\prime}\left(q=i \beta_{m}\right)}=\frac{\left.\cosh [q(1-\tilde{x})] e^{q^{2} \tilde{t}}\right|_{q=i \beta_{m}}}{\left.(d D(q) / d s)\right|_{q=i \beta_{m}}}
$$

where $D(q)=q^{2}\left[P q^{2} \cosh (q)+q\left(P \widetilde{R}_{c} q^{2}+1\right) \sinh (q)\right]$ and its derivative $d D(q) / d s$ may be calculated by using the chain rule as shown below.

$$
\frac{d D(q)}{d s}=\frac{d D(q)}{d q} \frac{d q}{d s}=\frac{1}{2 q} \frac{d D(q)}{d q}
$$

According to Eq. (A.6) the above derivative must be evaluated for $q=i \beta_{m}$. Thus, by using the eigencondition in the form $\sin (\beta)=-P \beta \cos (\beta) /\left(1-P \widetilde{R}_{c} \beta^{2}\right)$, after lengthy algebra, it results in

$$
\begin{aligned}
& \left.\frac{1}{2 q} \frac{d D}{d q}\right|_{q=i \beta_{m}}=\frac{1}{2}\left\{\left[P \widetilde{R}_{c} \beta_{m}^{4}-(4 P+1) \beta_{m}^{2}\right] \cos \left(\beta_{m}\right)\right. \\
& \left.+\left[\left(1+5 \widetilde{R}_{c}\right) P \beta_{m}^{3}-3 \beta_{m}\right] \sin \left(\beta_{m}\right)\right\} \\
& =\frac{\left[\left(P \widetilde{R}_{c}\right)^{2} \beta_{m}^{4}+\left(P+P \widetilde{R}_{c}-2 \widetilde{R}_{c}\right) P \beta_{m}^{2}+P+1\right] \beta_{m}^{2} \cos \left(\beta_{m}\right)}{2\left(P \widetilde{R}_{c} \beta_{m}^{2}-1\right)}
\end{aligned}
$$

Therefore, by substituting the above equation into Eq. (A.6), bearing in mind Eq. (A.7), the same expression defined by Eq. (10b) is obtained.

\section{Nomenclature}

A: $\quad$ Counting integer for accuracy $\left(10^{-A}\right)$

$C: \quad$ Volumetric heat capacity $\left[\mathrm{kJ} / \mathrm{m}^{3 \circ} \mathrm{C}\right]$

$g_{\mathrm{f}, 0}$ : Volumetric heat generation $\left[\mathrm{W} / \mathrm{m}^{3}\right]$

$H(\cdot)$ : Heaviside or unit step function

$k$ : Thermal conductivity $\left[\mathrm{W} / \mathrm{m}^{\circ} \mathrm{C}\right]$

L: Thickness [m]

$\widetilde{N}_{m}:$ Dimensionless norm

$P: \quad$ Heat capacity ratio

$q$ : Auxiliary variable in the Laplace domain $(q=\sqrt{s})$

$q_{\mathrm{f}, 0}^{\prime \prime}$ : Applied heat flux per unit area $\left(q_{\mathrm{f}, 0}^{\prime \prime}=g_{\mathrm{f}, 0} L_{\mathrm{f}}\right)\left[\mathrm{W} / \mathrm{m}^{2}\right]$

$R: \quad$ Residue

$R_{c}$ : Thermal contact resistance $\left[\mathrm{m}^{2 \circ} \mathrm{C} / \mathrm{W}\right]$

$s: \quad$ Laplace variable

$t: \quad$ Time [s]

$t_{d}: \quad$ Deviation time $[\mathrm{s}]$

T: $\quad$ Temperature $\left[{ }^{\circ} \mathrm{C}\right]$

$x$ : Spatial coordinate $[\mathrm{m}]$

$X: \quad$ Scaled sensitivity coefficient $\left[{ }^{\circ} \mathrm{C}\right]$

$z_{m}$ : Initial guess for eigenvalue.

\section{Greek Symbols}

$\alpha$ : Thermal diffusivity, $k / C\left[\mathrm{~m}^{2} / \mathrm{s}\right]$

$\beta_{m}: m$-th exact eigenvalue

$\varepsilon$ : Error for the computational solution

$\zeta_{m}$ : Approximate eigenvalue

Э: Temperature in the Laplace domain

$\sigma$ : Standard deviation of measurement errors $\left[{ }^{\circ} \mathrm{C}\right]$

$\sigma_{k}:$ Standard deviation of $k\left[\mathrm{~W} / \mathrm{m}^{\circ} \mathrm{C}\right]$

$\sigma_{C}:$ Standard deviation of $C\left[\mathrm{~kJ} / \mathrm{m}^{3 \circ} \mathrm{C}\right]$

$\varphi$ : $\quad$ Phase in the Laplace domain

Superscripts

: Dimensionless.

Subscripts

in: Initial

$f$ : Surface layer or film (heater)

$m$ : Counting integer for eigenvalues.

\section{Data Availability}

The data used to support the findings of this study are included within the article.

\section{Conflicts of Interest}

The authors declare that there are no conflicts of interest regarding the publication of this paper.

\section{References}

[1] R. L. McMasters and J. V. Beck, "Using derivative regularization in parameter estimation," Inverse Problems in Science and Engineering, vol. 8, no. 4, pp. 365-390, 2000.

[2] K. J. Dowding, B. F. Blackwell, and R. J. Cochran, "Application of sensitivity coefficients for heat conduction problems," Numerical Heat Transfer, Part B: Fundamentals, vol. 36, no. 1, pp. 33-55, 1999.

[3] B. Blackwell and J. V. Beck, "A technique for uncertainty analysis for inverse heat conduction problems," International Journal of Heat and Mass Transfer, vol. 53, no. 4, pp. 753-759, 2010.

[4] H. Molavi, A. Hakkaki-Fard, R. K. Rahmani, A. Ayasoufi, and M. Molavi, "A novel methodology for combined parameter and function estimation problems," Journal of Heat Transfer, vol.132, no. 12, pp. 1-11, 2010.

[5] C. Aviles-Ramos and A. Haji-Sheikh, "Estimation of thermophysical properties of composites using multi-parameter estimation and zeroth-order regularization," Inverse Problems in Science and Engineering, vol. 9, no. 5, pp. 507-536, 2001.

[6] A. Sakly, A. Jemni, P. Lagonotte, and D. Petit, "Estimation of the effective thermal properties in a metallic medium by an inverse technique using temperatures measurements," Journal of Applied Fluid Mechanics, vol. 4, no. 4, pp. 23-29, 2011.

[7] M. Cui, X. Gao, and J. Zhang, "A new approach for the estimation of temperature-dependent thermal properties by solving transient inverse heat conduction problems," International Journal of Thermal Sciences, vol. 58, pp. 113-119, 2012. 
[8] J. V. Beck and K. J. Arnold, Parameter Estimation in Engineering and Science, John Wiley \& Sons, New York, NY, USA, 1977.

[9] D. K. Mishra, K. D. Dolan, J. V. Beck, and F. Ozadali, "A novel instrument for rapid measurement of temperature-dependent thermal properties of conduction-heated food up to $140 \circ \mathrm{C}$," Journal of Food Engineering, vol. 191, pp. 19-27, 2016.

[10] H. S. Carslaw and J. C. Jaeger, Conduction of Heat in Solids, Oxford University Press, New York, NY, USA, 2nd edition, 1959.

[11] M. A. Al-Nimr and M. K. Alkam, "A generalized thermal boundary condition," Heat and Mass Transfer, vol. 33, no. 1-2, pp. 157-161, 1997.

[12] K. D. Cole, J. V. Beck, A. Haji-Sheikh, and B. Litkouhi, Heat Conduction Using Greens Function, CRC Press Taylor \& Francis, Boca Raton, FL, USA, 2nd edition, 2011.

[13] K. D. Cole, J. V. Beck, K. A. Woodbury, and F. De Monte, "Intrinsic verification and a heat conduction database," International Journal of Thermal Sciences, vol. 78, pp. 36-47, 2014.

[14] V. S. Arpaci, Conduction Heat Transfer, Addison-Wesley Publishing Company, Reading, MA, USA, 1966.

[15] A. Haji-Sheikh and J. V. Beck, "An efficient method of computing eigenvalues in heat conduction," Numerical Heat Transfer, Part B: Fundamentals, vol. 38, no. 2, pp. 133-156, 2000.

[16] G. D’Alessandro, F. de Monte, and D. E. Amos, "X62B10T00, Exact Analytical Conduction Toolbox," 2017, http://www.exact .unl.edu/exact/home/home.php.

[17] K. Oldham, J. Myland, and J. Spanier, An Atlas of Functions, Springer, New York, NY, USA, 2nd edition, 2009.

[18] F. De Monte, J. V. Beck, and D. E. Amos, "Solving twodimensional Cartesian unsteady heat conduction problems for small values of the time," International Journal of Thermal Sciences, vol. 60, pp. 106-113, 2012.

[19] G. D'Alessandro and F. De Monte, "Intrinsic verification of an exact analytical solution in transient heat conduction," Computational Thermal Sciences, vol. 10, no. 3, pp. 251-272, 2018.

[20] B. F. Blackwell, K. J. Dowding, and R. J. Cochran, "Development and implementation of sensitivity coefficient equations for heat conduction problems," Numerical Heat Transfer, Part B: Fundamentals, vol. 36, no. 1, pp. 15-32, 1999.

[21] F. de Monte and J. V. Beck, "X22B10T0, Exact Analytical Conduction Toolbox," 2013, http://www.exact.unl.edu/exact/home/ home.php.

[22] G. D'Alessandro and F. de Monte, "X42B10T00, Exact Analytical Conduction Toolbox," 2016, http://www.exact.unl.edu/exact/ home/home.php.

[23] G. D’Alessandro and F. de Monte, "Sensitivity coefficients for thermal property measurements using a boundary condition of the 4th kind," Journal of Physics: Conference Series, vol. 796, no. 1, article 012011, 2017. 


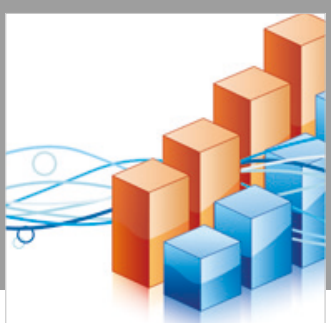

Advances in

Operations Research

\section{-n-m}
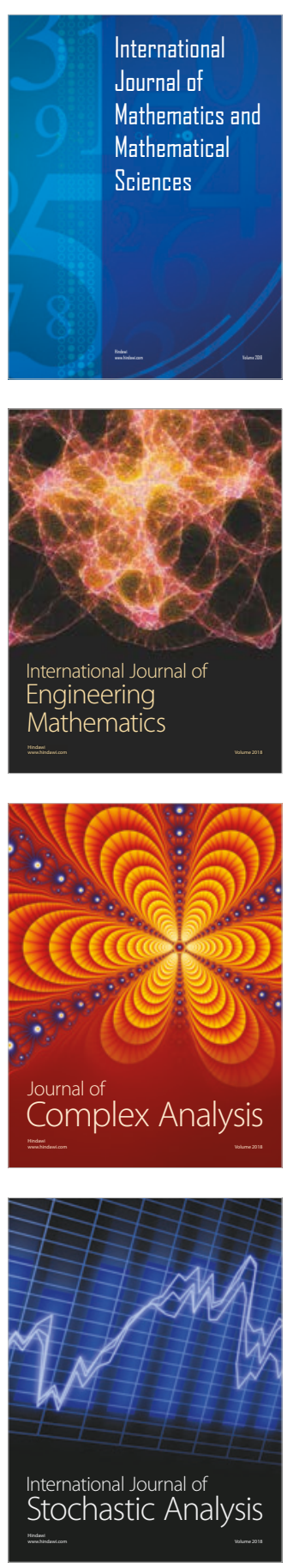
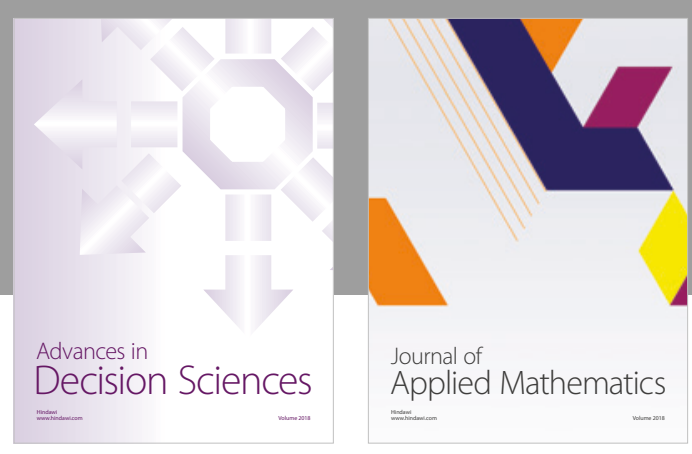

Journal of

Applied Mathematics
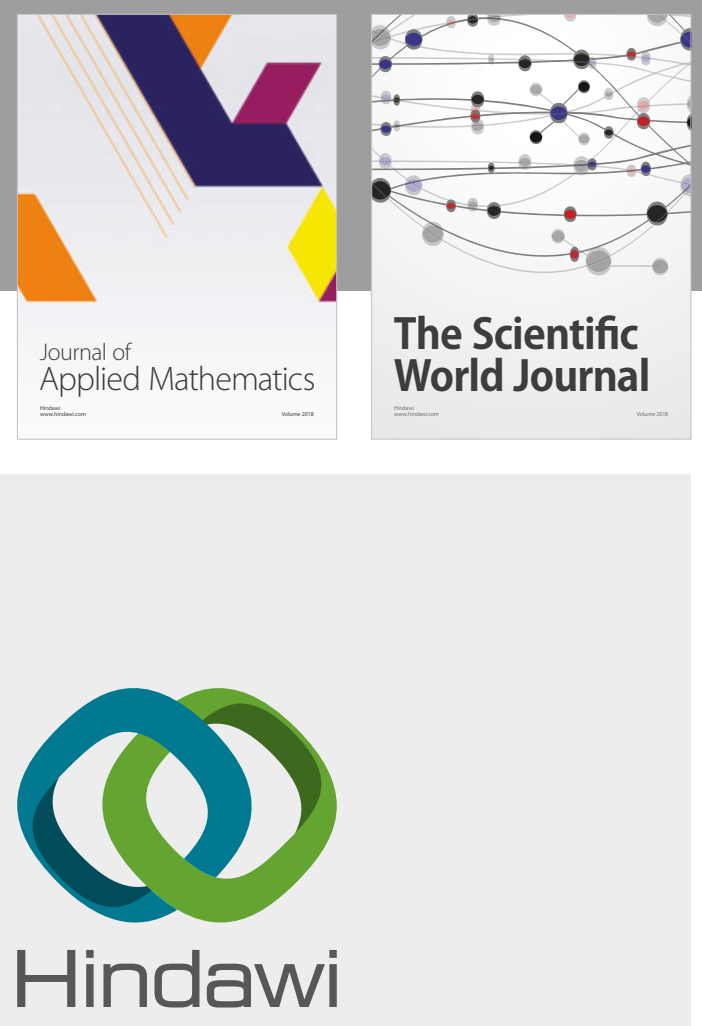

Submit your manuscripts at

www.hindawi.com

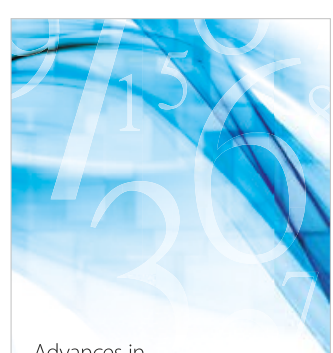

Advances in
Numerical Analysis
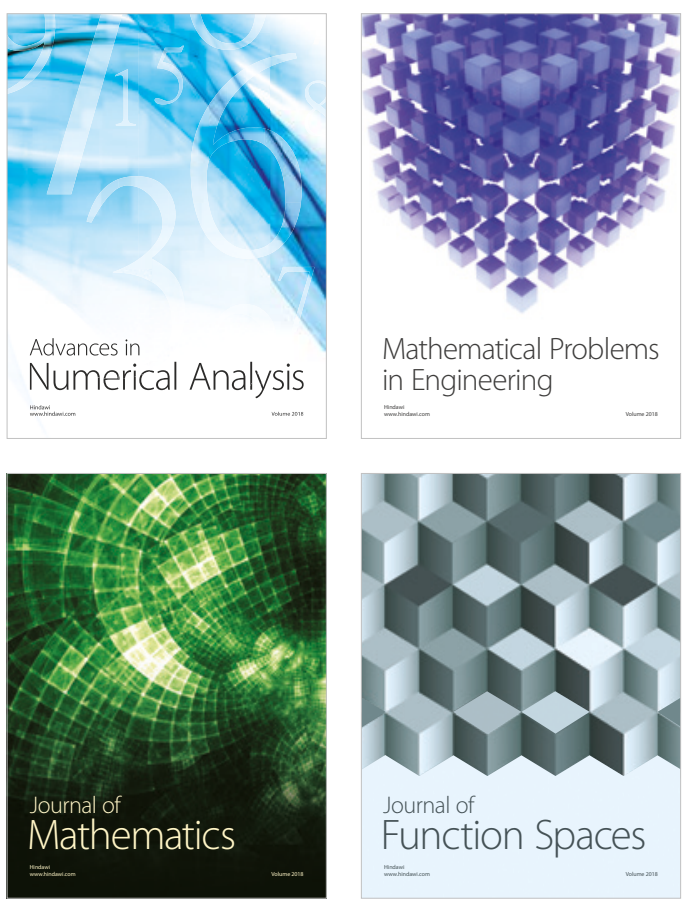

Mathematical Problems in Engineering

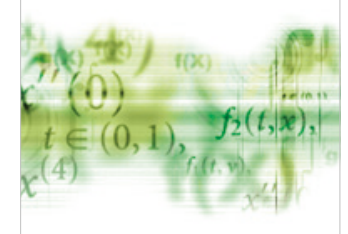

International Journal of

Differential Equations

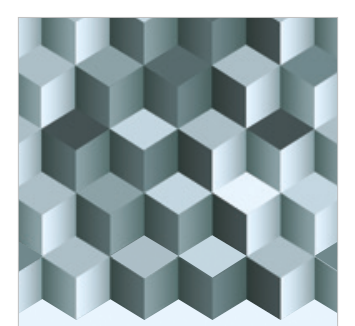

Journal of

Function Spaces
The Scientific

World Journal

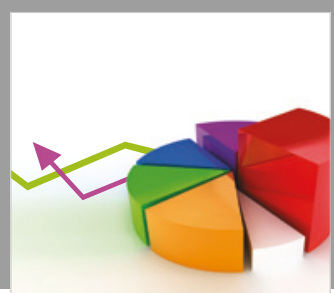

Journal of

Probability and Statistics
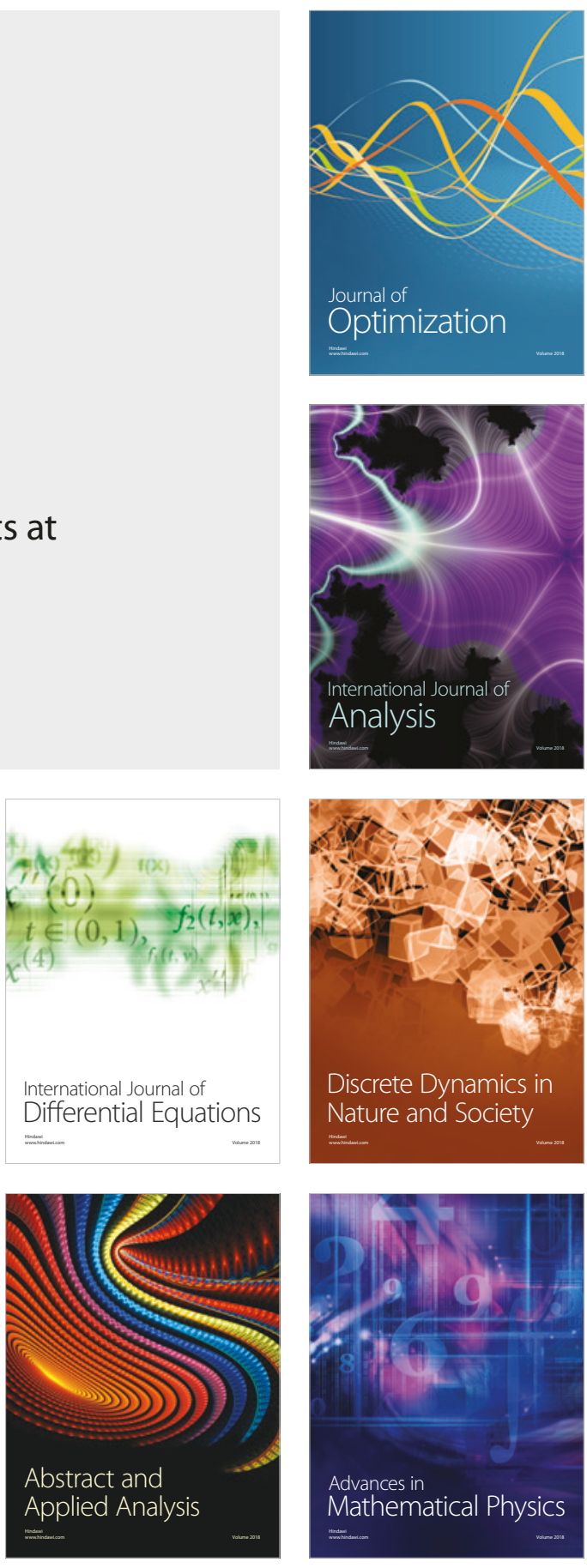